\title{
OCORRÊNCIA E ATIVIDADE BIOLÓGICA DE QUASSINÓIDES DA ÚLTIMA DÉCADA
}

\author{
Maria Mozarina Beserra Almeida* \\ Departamento de Química Orgânica e Inorgânica, Departamento de Química Analítica e Físico-Química, Universidade Federal \\ do Ceará, CP 12200, 60451-970 Fortaleza - CE, Brasil \\ Ângela Martha Campos Arriaga, Allana Kellen Lima dos Santos e Telma L. G. Lemos \\ Departamento de Química Orgânica e Inorgânica, Universidade Federal do Ceará, CP 12200, 60451-970 Fortaleza - CE, Brasil \\ Raimundo Braz-Filho e Ivo J. Curcino Vieira \\ Setor de Química de Produtos Naturais, Universidade Estadual do Norte Fluminense Darcy Ribeiro, Av. Alberto Lamego, 2000, \\ 28013-600 Rio de Janeiro - RJ, Brasil
}

Recebido em 13/1/06; aceito em 9/8/06; publicado na web em 27/4/07

\begin{abstract}
OCCURRENCE AND BIOLOGICAL ACTIVITY OF QUASSINOIDS IN THE LAST DECADE. Quassinoids, the bitter principles of the Simaroubaceae family, are a group of structurally complex and highly oxygenated degraded triterpenes. They are divided into five groups according to their basic skeleton, C-18, C-19, C-20, C-22 and C-25. In recent years, attention has been focused on quassinoids because several of them have shown promising biological activities. This paper features a review of some characteristics of these compounds, updating known information with discoveries from the last decade and covering chemical structures, basic skeletons, occurrence in genus and species, besides detailed studies of biological activities such as antitumor, antimalarial, phytotoxic, antifeedant, insecticidal, anti-inflammatory and antiulcer.
\end{abstract}

Keywords: quassinoid; molecular structure; biological activities.

\section{INTRODUÇÃO}

Os quassinóides constituem uma classe de substâncias encontradas quase que exclusivamente em plantas da família Simaroubaceae e, quimicamente são considerados triterpenos biodegradados com alto padrão de oxigenação, apresentando uma ampla faixa de atividade biológica ${ }^{1}$.

A existência de substâncias com sabor amargo na madeira da espécie Quassia amara, nativa das Guianas Francesas, foi primeiramente registrada na literatura em 1835 e, como a maioria das substâncias naturais isoladas de plantas no século XIX, só teve sua estrutura elucidada muitos anos mais tarde, em função do desenvolvimento de técnicas experimentais de análise estrutural ${ }^{1}$. Em 1937 foi isolado o primeiro quassinóide e, somente em 1950, Robertson e colaboradores caracterizaram os constituintes químicos majoritários da Quassia amara, denominados de quassina (1) e neoquassina (2). Somente uma década mais tarde, após extensivos estudos, Valenta e colaboradores determinaram a estereoquímica completa desses compostos, sendo estes os primeiros quassinóides relatados na família Simaroubaceae ${ }^{2}$ (Figura 1).

$\mathrm{Na}$ década de 70, ensaios biológicos com quassinóides revelaram que possuíam uma acentuada atividade antileucêmica, permitindo já na década seguinte que o quassinóide bruceantina (3) (Figura 1) entrasse em uso clínico, nos Estados Unidos, na quimioterapia desse tipo de câncer ${ }^{1,3}$. Esse fato incentivou inúmeras pesquisas nas áreas de química e farmacologia com plantas da família Simaroubaceae, levando à descoberta de vários outros quassinóides bioativos, contribuindo para a ampliação do conhecimento dessas substâncias e, conseqüentemente, da química de produtos naturais. Isso pode ser constatado através de dados obtidos de levantamento bibliográfico, tendo como fonte de consulta principalmente os sites

\footnotetext{
*e-mail: mozaba@ufc.br
}
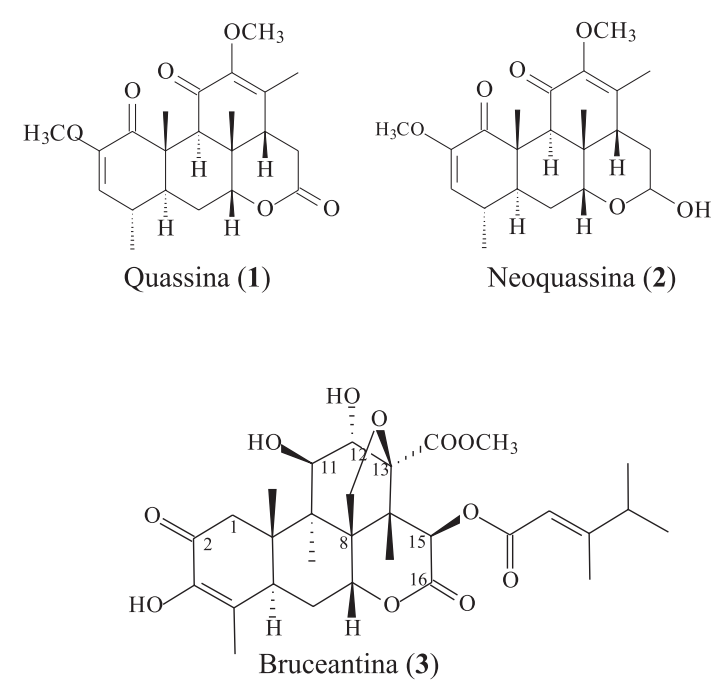

Figura 1. Primeiros quassinóides isolados (Quassina e Neoquassina) e estrutura química da Bruceantina. Reproduzida da ref. 2, com permissão da ACS

científicos de busca, "Web of Science", "Science@Direct" e Scielo (Figura 2), observando-se um aumento significativo de publicações sobre esta classe de substâncias entre os anos de 1960 e 2005, totalizando 502 trabalhos.

Estruturalmente, os quassinóides podem ser classificados em diferentes grupos, de acordo com seus esqueletos básicos (Figura 3): $\mathbf{C}_{18}, \mathbf{C}_{19}, \mathbf{C}_{20}, \mathbf{C}_{22}$ e $\mathbf{C}_{25}$, sendo que a grande maioria se apresenta com esqueleto básico do tipo $\mathbf{C}_{20}$, onde se inclui a quassina (1). Tais substâncias naturais são derivadas de triterpenos da série eufol/ tirucalol, em sua grande maioria altamente oxigenados, contendo lactonas em seus esqueletos básicos e, raramente, possuindo mais que uma ligação dupla. Apresentam diferentes grupos funcionais 


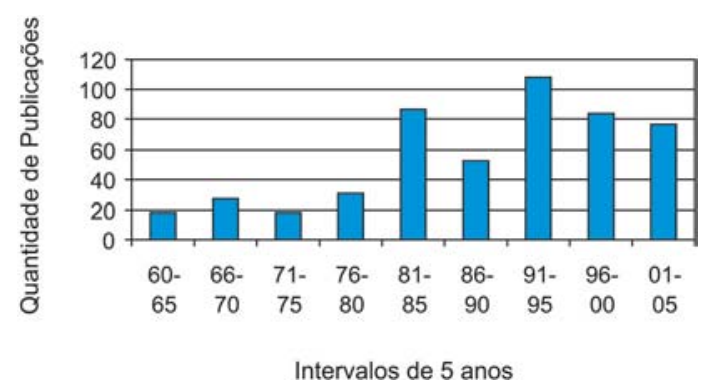

Figura 2. Número de publicações com quassinóides (1960-2005) por período de 5 anos. 60-65 significa o período de 1960 a 1965
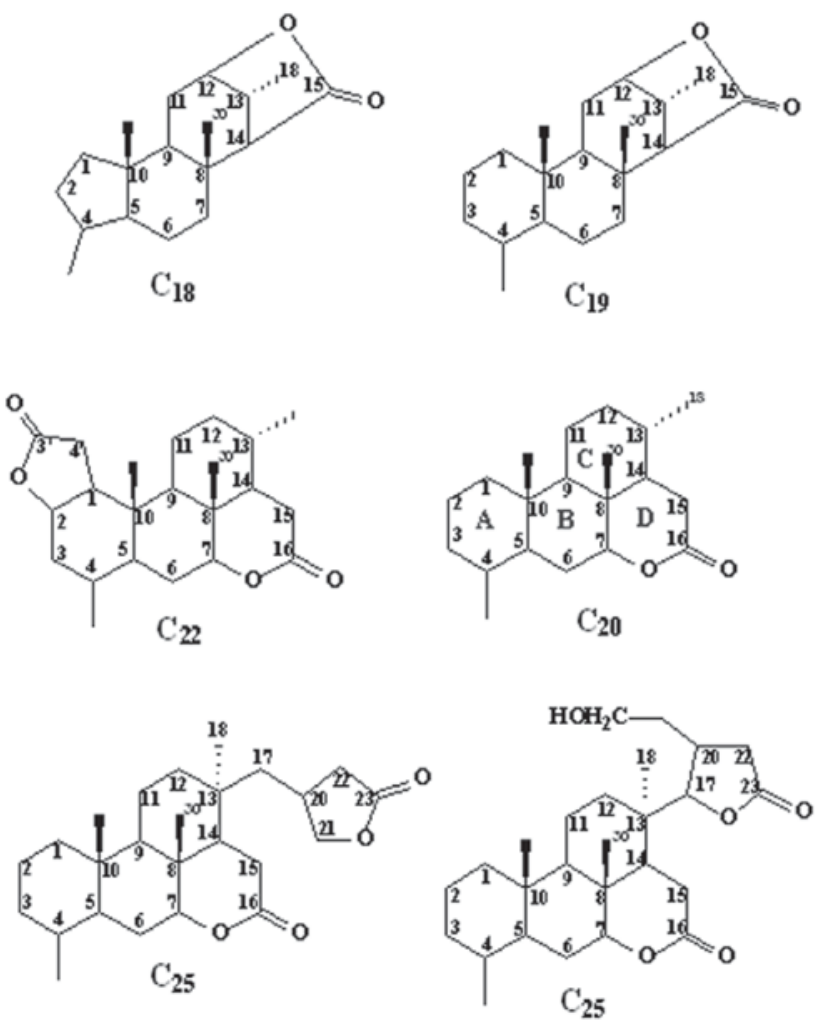

Figura 3. Esqueletos básicos dos quassinóides

oxigenados em seus esqueletos, com exceção das posições C-5, C9 e dos grupos metilas nas posições C-4 e C-10 ${ }^{1}$.

A elucidação estrutural dos quassinóides representa um desafio para os espectroscopistas, despertando interesse de muitos grupos de pesquisa em todo o mundo. A elucidação estrutural destas substâncias é caracterizada por dados espectrais, principalmente RMN 1D e 2D, para atribuição dos deslocamentos químicos dos átomos de carbono e hidrogênio ${ }^{4,5}$.

Este trabalho tem como objetivo contribuir para complementar e atualizar as duas revisões existentes publicadas até o momento sobre os quassinóides ${ }^{1,4}$, apresentando dados compilados sobre essas substâncias isoladas nos últimos 10 anos e aspectos importantes de suas diversas atividades biológicas.

\section{OCORRÊNCIA DOS QUASSINÓIDES}

A família Simaroubaceae é constituída por aproximadamente 32 gêneros e 200 espécies, distribuídas em todas as regiões tropicais e subtropicais do globo. No Brasil, está representada pelos gêneros Quassia e Picrolemma, na região Amazônica; Castela e
Picrasma, no sul do país; Simaba e Simarouba em quase todas as regiões brasileiras ${ }^{6}$.

As Simaroubaceae geralmente se apresentam como árvores ou arbustos, tendo como característica marcante um sabor bastante amargo em seu córtex. Assim, muitas espécies dessa família (Quassia amara, Picrasma excelsa, Jamaica quassia) são conhecidas há mais de um século, por conterem substâncias amargas, denominadas de "quassina", nome emprestado a toda esta classe de compostos estruturalmente relacionados, denominados de quassinóides ${ }^{4}$. Estes compreendem um grupo de substâncias naturais quase que exclusivo de espécies das Simaroubaceae, podendo, assim, ser considerado marcador taxonômico desta família ${ }^{7,8}$. A presença ou ausência de quassinóides levou alguns gêneros a serem excluídos da família, sendo este um importante parâmetro de classificação taxonômica de Simaroubaceae. Um exemplo é o gênero Picramnia, excluído das Simaroubaceae em 1995 e incluído em uma nova família chamada Picramniaceae, após alguns estudos fitoquímicos ${ }^{9}$.

A atualização bibliográfica sobre a ocorrência dos quassinóides dos últimos 10 anos (1994 a 2005) resultou na Tabela 1, onde constam espécies, estruturas químicas, nomes vulgares e referências. Esta tabela apresenta os quassinóides isolados das espécies de Simaroubaceae, perfazendo um total de 96 compostos.

A Figura 4 mostra, para o período mencionado, os percentuais de ocorrência de quassinóides nos 9 gêneros de Simaroubaceae em que foram encontrados. Observa-se que, dentre as espécies estudadas, o maior registro de quassinóides está no gênero Brucea com 20,8\%, seguido de Ailanthus (18,8\%), Eurycoma (13,5\%), Quassia (12,5\%), Castela e Picrasma (10,4\%), Simaba (7,3\%), Simarouba $(4,2 \%)$ e Picrolemma $(2,1 \%)$. A Figura 5 evidencia o percentual de ocorrência de esqueletos básicos dessas substâncias, verificandose maior incidência de compostos $\mathrm{C}_{20}(76,1 \%)$, superior à dos demais, que ocorreram em menores proporções: $\mathrm{C}_{19}(18,8 \%), \mathrm{C}_{18}$

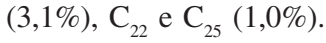

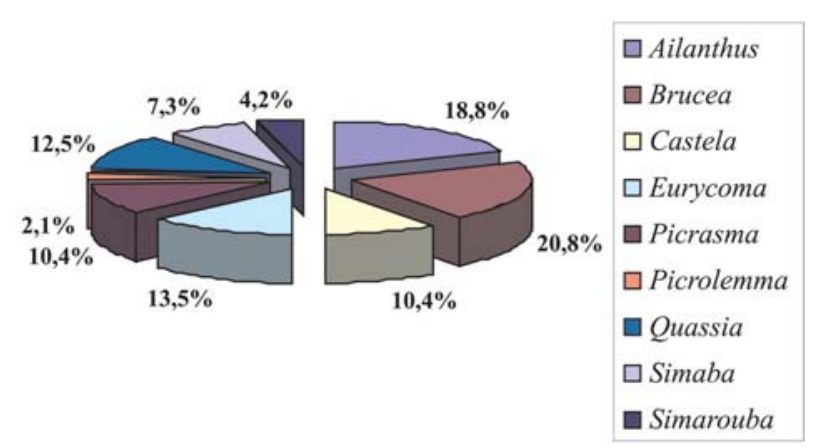

Figura 4. Ocorrência dos quassinóides nos gêneros de Simaroubaceae

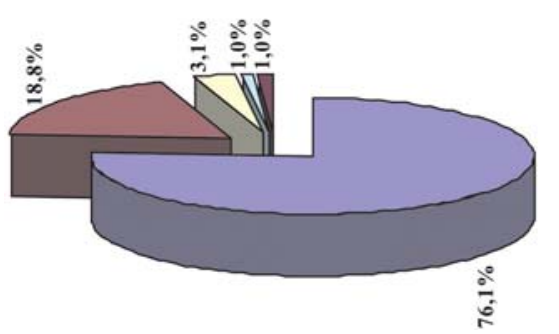

$\square \mathrm{C} 20$

$\square \mathrm{C} 19$

$\square \mathrm{C} 18$

$\square \mathrm{C} 22$

$\square \mathrm{C} 25$

Figura 5. Ocorrência dos esqueletos básicos dos quassinóides em Simaroubaceae 
Tabela 1. Quassinóides isolados nas espécies de Simaroubaceae (1994-2005)

Espécies Substâncias / Nomes Vulgares

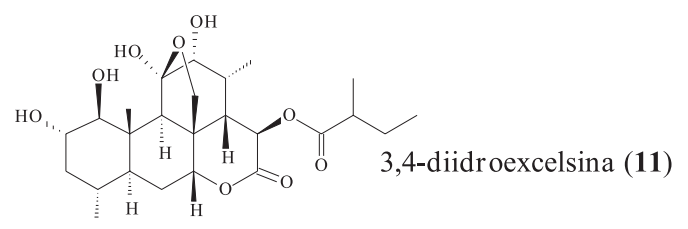

Ailanthus excelsa
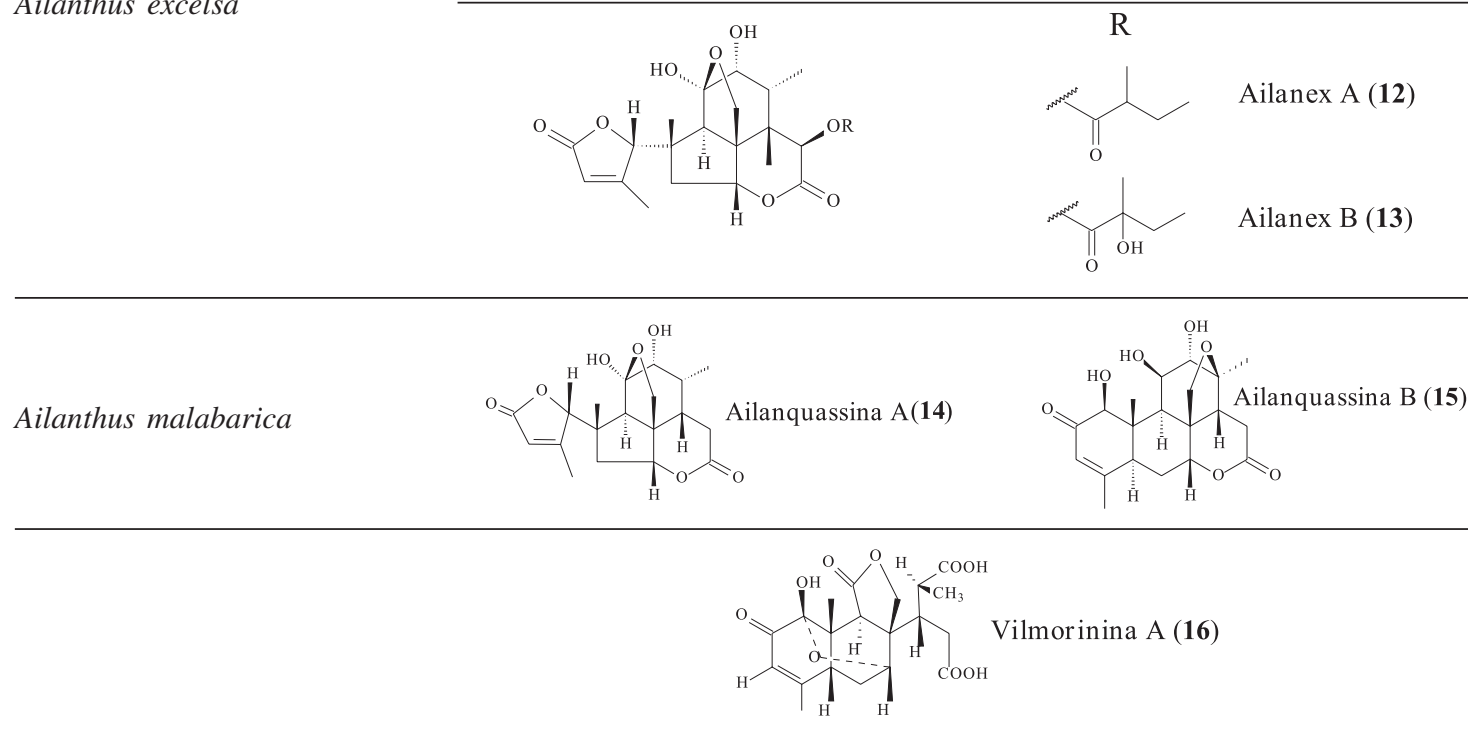

Ailanthus vilmoriniana

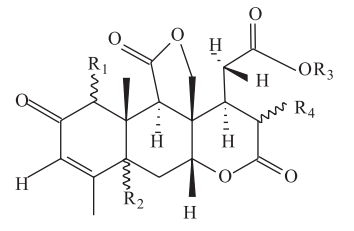

$\begin{array}{ccccc}\mathrm{R}_{1} & \mathrm{R}_{2} & \mathrm{R}_{3} & \mathrm{R}_{4} & \\ \beta-\mathrm{OH} & \alpha-\mathrm{H} & \mathrm{CH}_{3} & \beta-\mathrm{CH}_{3} & \text { Vilmorinina B (17) } \\ \beta-\mathrm{OH} & \alpha-\mathrm{H} & \mathrm{H} & \beta-\mathrm{CH}_{3} & \text { Vilmorinina C (18) } \\ \alpha-\mathrm{OH} & \alpha-\mathrm{H} & \mathrm{H} & \beta-\mathrm{CH}_{3} & \text { Vilmorinina D (19) } \\ \beta-\mathrm{OH} & \beta-\mathrm{H} & \mathrm{H} & \beta-\mathrm{CH}_{3} & \text { Vilmorinina E (20) } \\ \beta-\mathrm{OH} & \beta-\mathrm{H} & \mathrm{H} & \alpha-\mathrm{CH}_{3} & \text { Vilmorinina F (21) }\end{array}$


Tabela 1. (continuação)

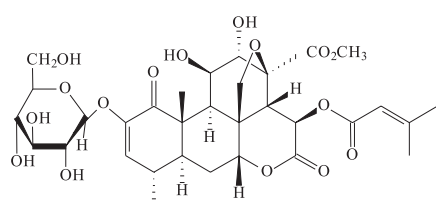

Javanicosida I (22)

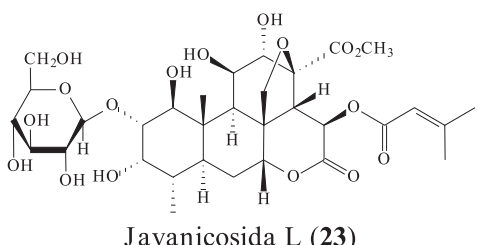

Javanicosida L (23)

Brucea amaríssima

$\begin{array}{lll}\mathrm{R}_{1} & \mathrm{R}_{2} & \mathrm{R}_{3}\end{array}$

$\mathrm{R}_{4}$
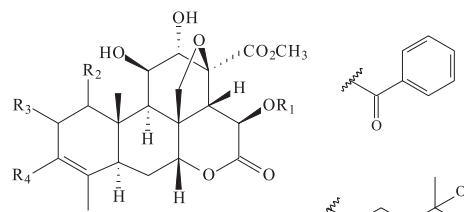

$\mathrm{H} \quad=\mathrm{O}$

$\mathrm{OH}_{\mathrm{OH}}^{\mathrm{O}} \mathrm{CH}_{2} \mathrm{OH}$

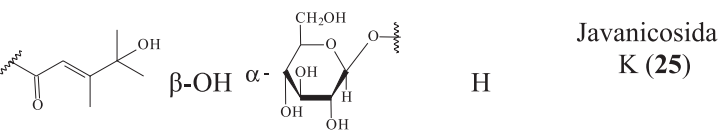

Brucea antidysenterica

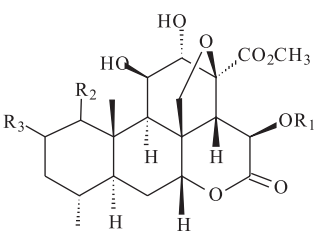

$\mathrm{R}_{1}$

$\mathrm{R}_{2} \quad \mathrm{R}_{3}$

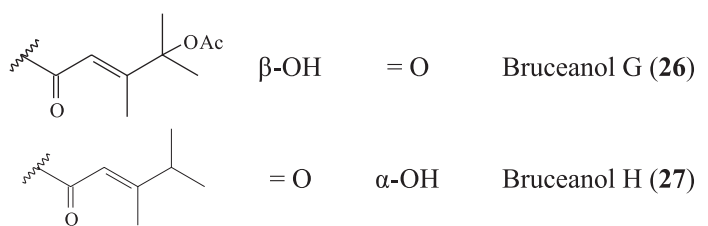
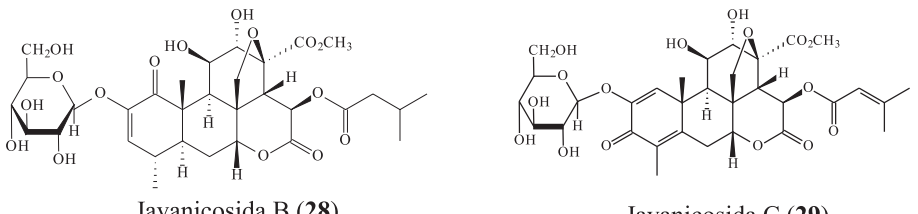

Javanicosida B (28)

Javanicosida C (29)

$\mathrm{R}$
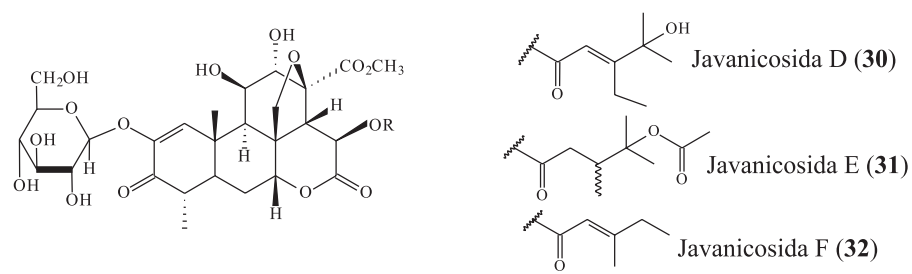

Brucea javanica
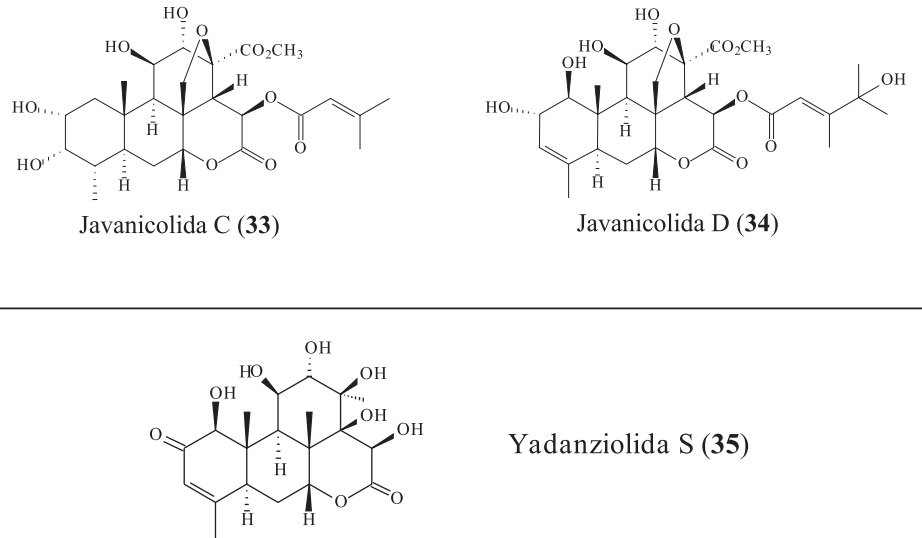
Tabela 1. (continuação)

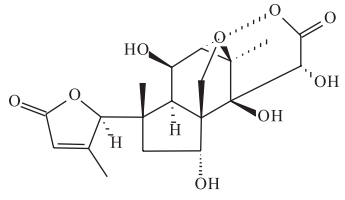

Javanicolida A (36)

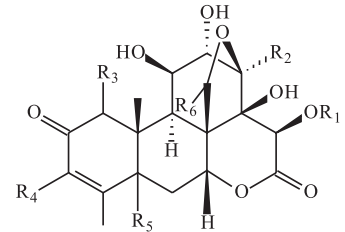

$\mathrm{R}_{5}$

$\mathrm{R}_{6}$

Brucea javanica

\begin{tabular}{|c|c|c|c|c|c|c|}
\hline $\mathrm{R}_{1}$ & $\mathrm{R}_{2}$ & $\mathrm{R}_{3}$ & $\mathrm{R}_{4}$ & $\mathrm{R}_{5}$ & $\mathrm{R}_{6}$ & \\
\hline $\mathrm{OH}$ & $\mathrm{CH}_{3}$ & $\beta-\mathrm{OH}$ & $\mathrm{H}$ & $\alpha-H$ & $\mathrm{OH}$ & Javanicolida B (37) \\
\hline O & $-\mathrm{CO}_{2} \mathrm{CH}_{3}$ & $\mathrm{H}$ & & $\beta-\mathrm{H}$ & $\mathrm{H}$ & Javanicosida A (38) \\
\hline
\end{tabular}

Castela peninsularis

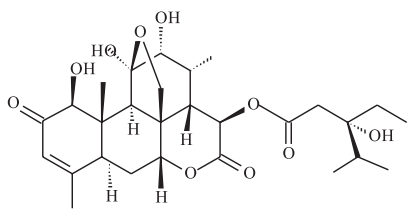

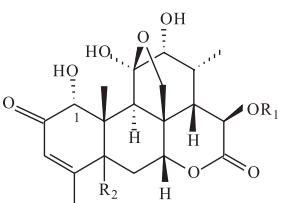

$\begin{array}{ll}\mathrm{R}_{1} & \mathrm{R}_{2} \\ \mathrm{Ac} & \alpha-\mathrm{H} \\ \mathrm{H} & \alpha-\mathrm{H} \\ \mathrm{H} & \beta-\mathrm{H}\end{array}$

$\mathrm{R}_{2}$

$-\mathrm{H}$

1-epi-holocantona (43)

1-epi-glaucarubolona (44)

1-epi-5-iso-glaucarubolona (45)

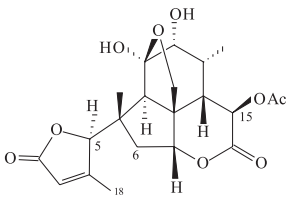

15-O-acetil-5(S)-poliandrol (46)
$\mathrm{R}$ glaucarubol (47)

$\Delta^{4,5}$-glaucarubol (48)
$15-O$-acetil $-\Delta^{4,5}-$

Castela polyandra

$$
\text { (49) }
$$

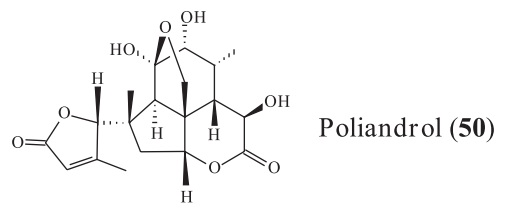


Tabela 1. (continuação)

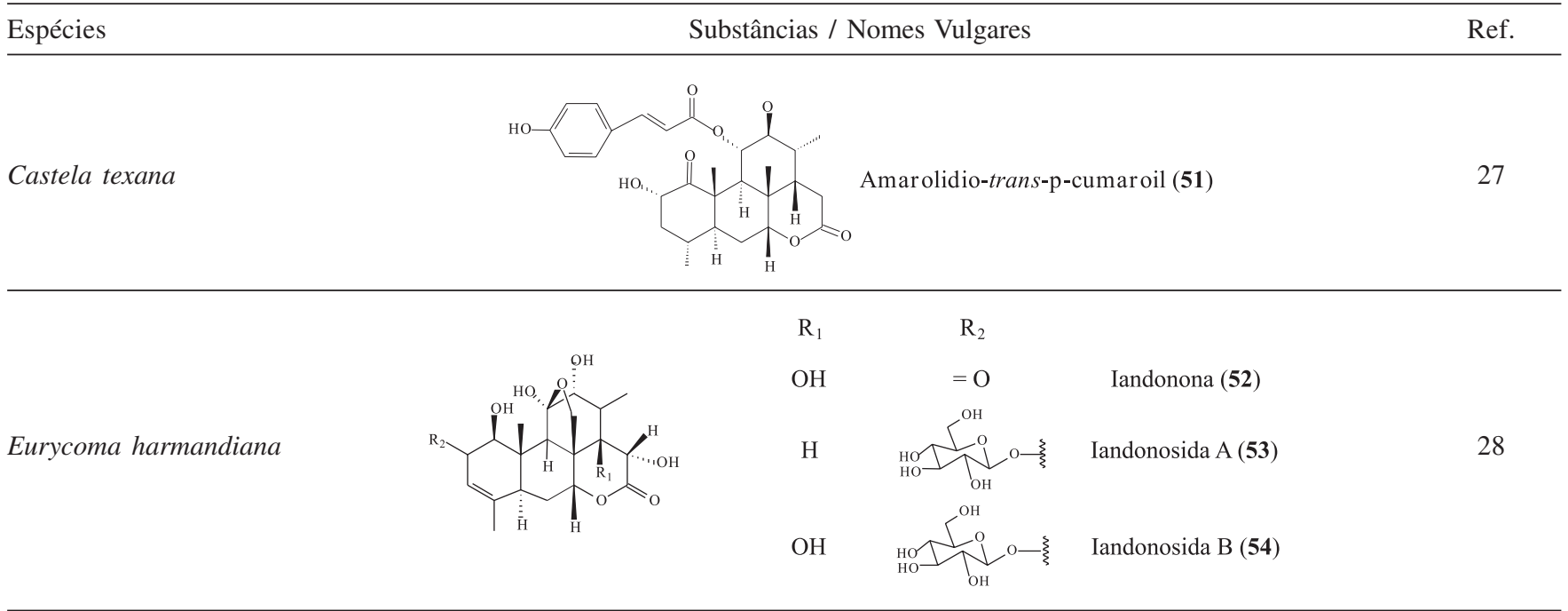

$$
\text { 12-epi-11-diidroclaineanona (55) }
$$

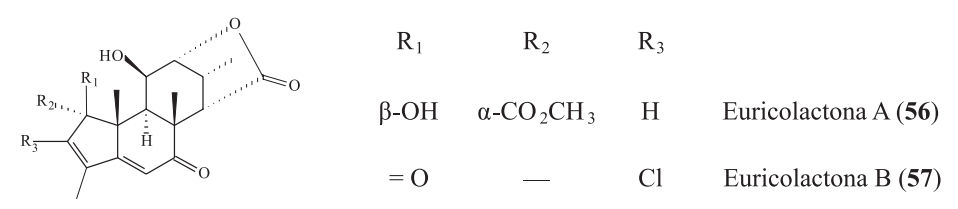

Eurycoma longifolia

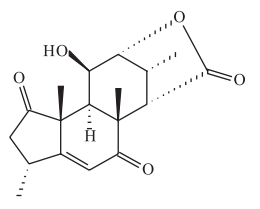

Euricolactona D (59)

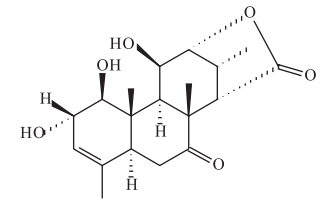

Euricolactona E (60)

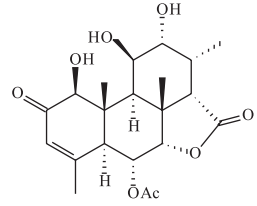

Euricolactona F (61)
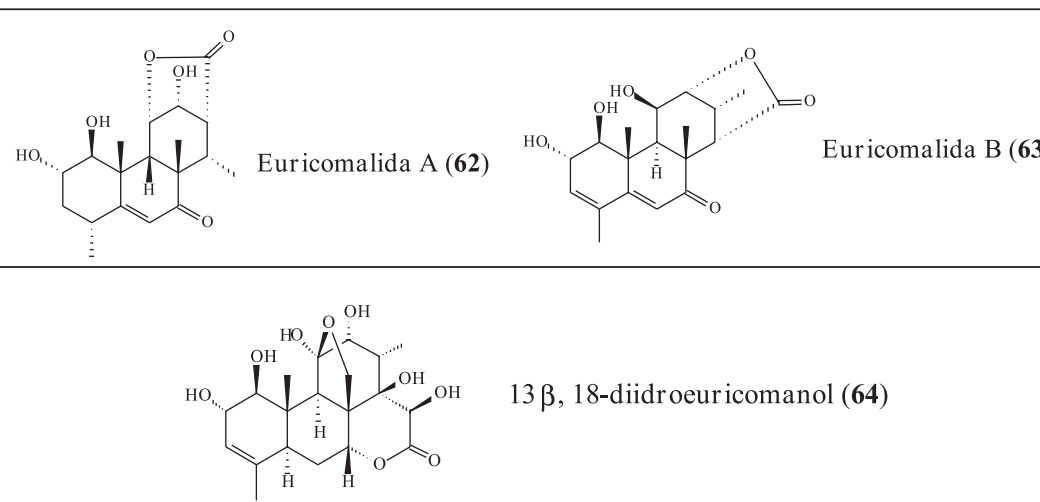

Picrasma ailanthoides

$$
\text { Picrasinol D (65) }
$$


Tabela 1. (continuação)

Espécies Picrasma javanica

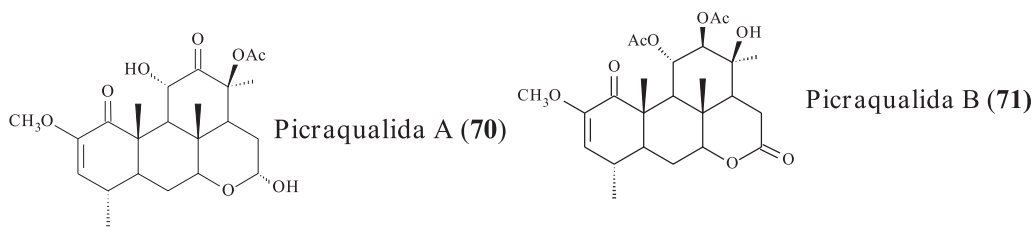

Picrasma quassioides

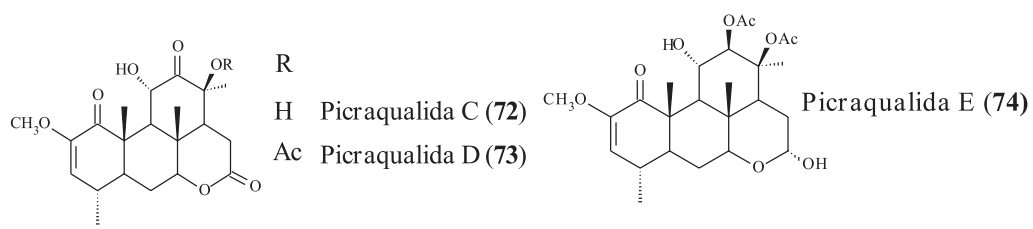

Picrolemma pseudocoffea

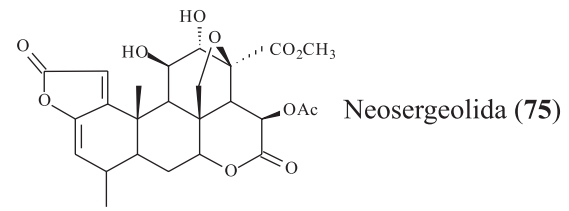

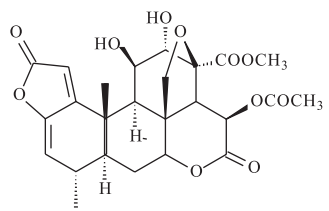

Picrolemma sprucei

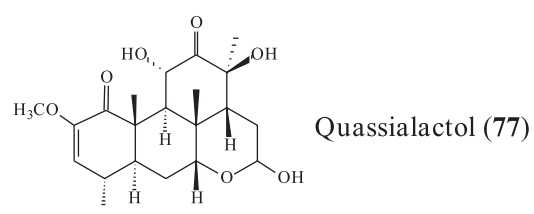

Quassia amara

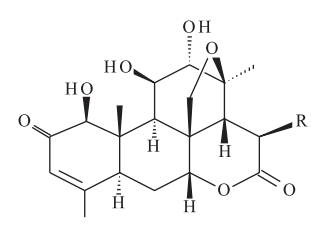

Quassia indica
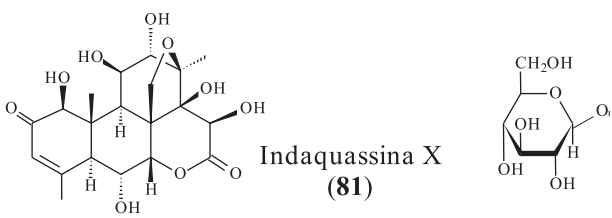
$\mathrm{R}$

$\begin{array}{cc}\text { OAc } & \text { Samaderina X (78) } \\ \text { H } & \text { Samaderina Y (79) } \\ \text { OH } & \text { Samaderina Z (80) }\end{array}$

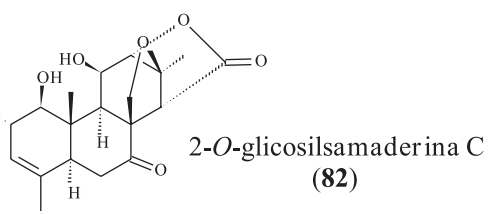


Tabela 1. (continuação)

Quassia indica
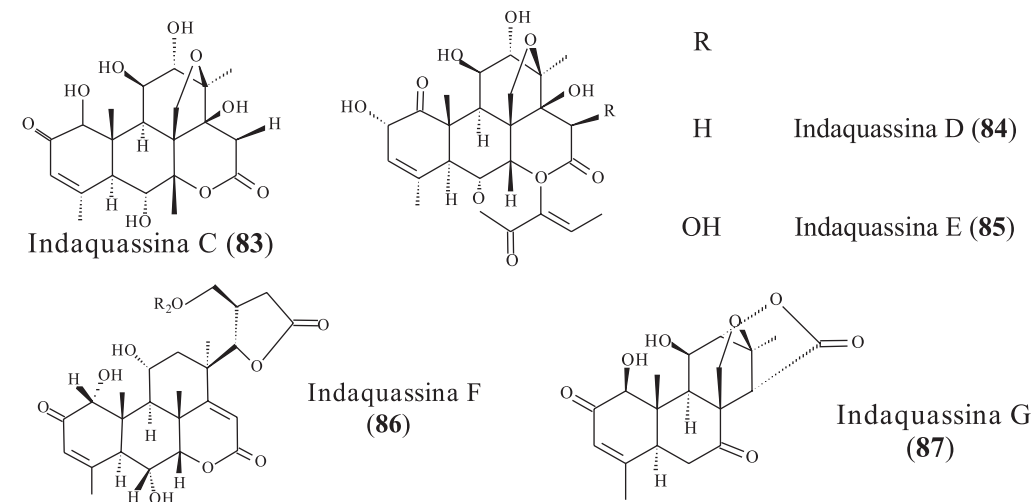

Quassia sp. aff. bidwillii
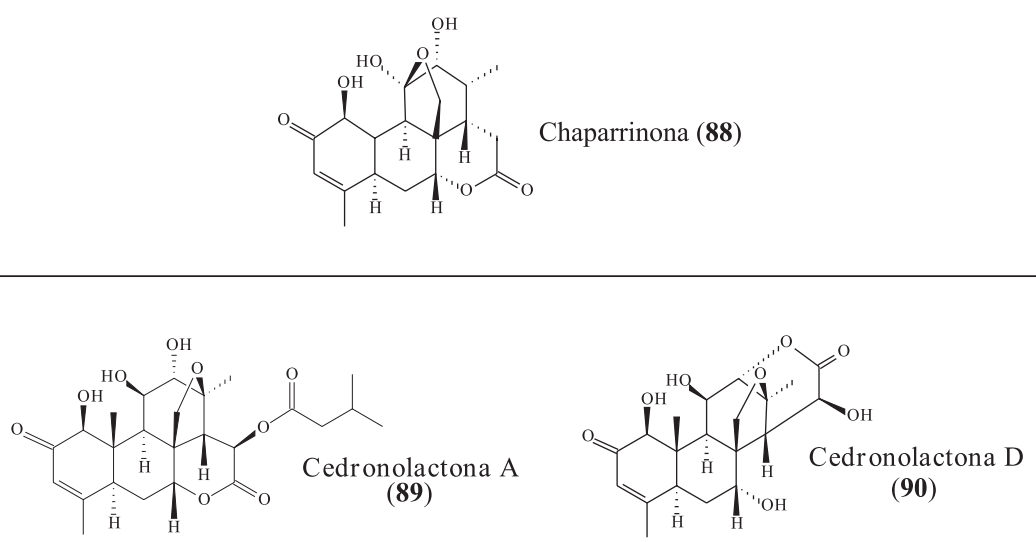

Simaba cedron

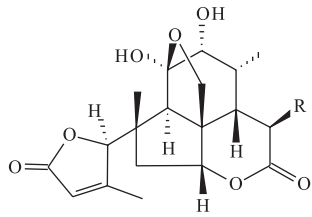

$\mathrm{R}$

H Cedronolactona B (91)

$\mathrm{OH}$

Cedronolactona C (92)

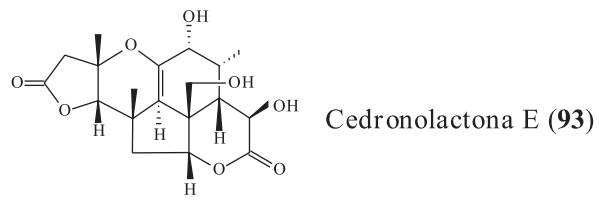

Simaba orinocensis

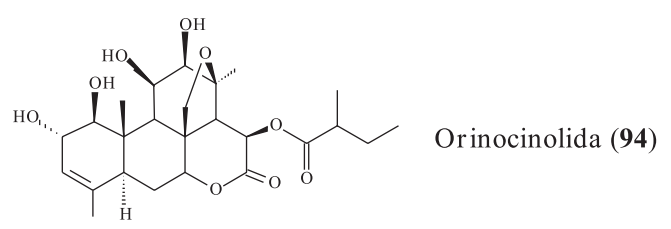


Tabela 1. (continuação)

Simarouba versicolor

Quando se compara a ocorrência dos esqueletos básicos nas diversas espécies (Figura 6), comprova-se a predominância do esqueleto $\mathrm{C}_{20}$, uma vez que, de 25 espécies estudadas, 16 delas $(A$. altissima, A. vilmoriniana, B. amarissima, B. antidysenterica, $C$. peninsulares, $C$. texana, E. harmandiana, $P$. ailanthoides, $P$. javanica, $P$. crenata, $P$. quassioides, $P$. pseudocoffea, $Q$. amara, $Q$. sp. aff. bidwillii, S. orinocensis, S. versicolor) apresentaram somente este tipo estrutural. $\mathrm{O}$ esqueleto $\mathrm{C}_{19}$ aparece em um número bem menor de espécies, destacando-se em ordem decrescente de ocorrência, principalmente, as espécies $S$. subcymosa (100\%), $S$. cedron (80\%), A. excelsa (66,7\%), E. longifolia e A. malabarica (50\%). A ocorrência de $\mathrm{C}_{18}, \mathrm{C}_{22} \mathrm{e}_{25}$ apresenta-se consideravelmente reduzida nas espécies estudadas, já que o esqueleto $\mathrm{C}_{18}$ aparece somente em E. longifolia; $\mathrm{C}_{22}$ é evidenciado apenas em $P$. sprucei e compostos $\mathrm{C}_{25}$ ocorrem somente na $Q$. indica.

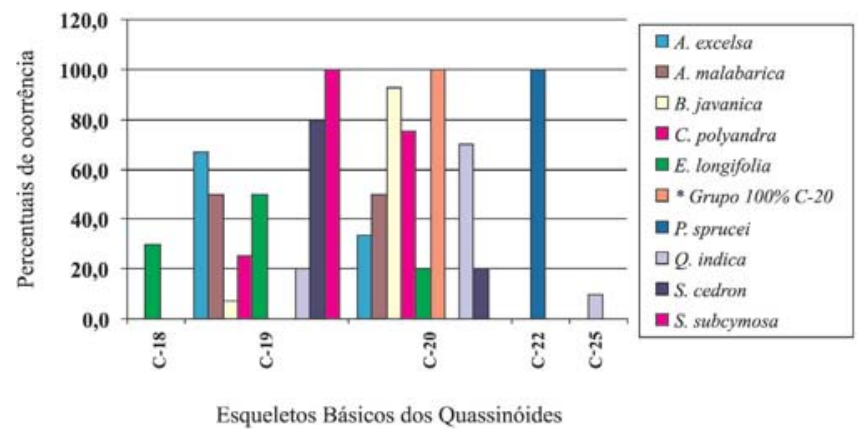

"Grupo: A. altissima, A. vilmoriniana, B. amarissima, B. antidysenterica, C. Peninsularis, C. texana, E harmandiana, $P$. ailanthoides, $P$ crenata, $P$. javanica, $P$. pseudocoffea, $P$. quassioides, $Q$ amara, $Q$. sp. aff.
bidwilli, S. orinocensis, $S$, versicolor

Figura 6. Ocorrência dos esqueletos básicos dos quassinóides nas espécies de Simaroubaceae

Outra constatação interessante nesse levantamento foi a ocorrência do quassinóide cedifilina (101) na espécie Cedrelopsis grevei
(Ptaeroxylaceae $)^{50}$, bem como da quassimarina (102) e da simalikalactona D (103) na Leitneria floridana (Leitneriaceae) ${ }^{51}$, os quais foram os primeiros registros fora da família Simaroubaceae (Figura 7).

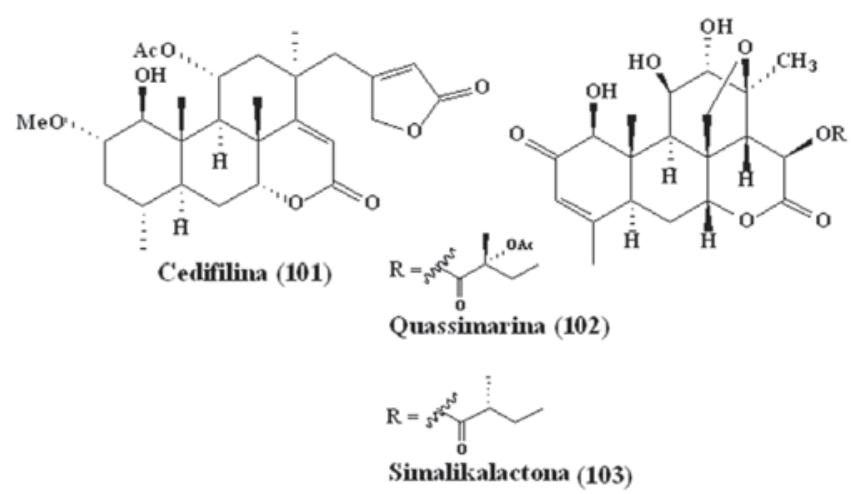

Figura 7. Estrutura química da cedifilina (101) [Reproduzida da ref. 50, com permissão da Elsevier], da quassimarina (102) e da simalikalactona (103), reproduzida da ref. 51, com permissão da ACS

\section{ASPECTOS BIOLÓGICOS}

Os quassinóides apresentam uma ampla faixa de atividade biológica, o que tem despertado grande interesse da comunidade científica. Plantas das Simaroubaceae são utilizadas há bastante tempo na medicina tradicional, com diversas finalidades, e estudos científicos provaram que a maior parte destas atividades se deve à presença dessa classe de substâncias ${ }^{7}$.

Nas próximas seções, faz-se um relato do potencial biológico avaliado e comprovado dessas substâncias, as quais têm suas estruturas apresentadas na Figura 8. Dados de atividade antineoplásica e antimalárica (registrados nos últimos 10 anos) encontram-se na Tabela 2. 


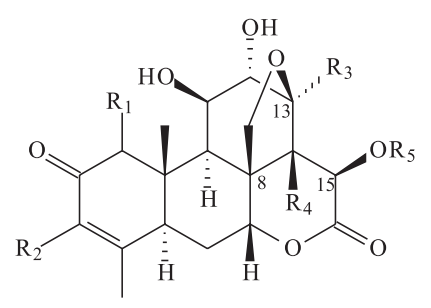

$\begin{array}{lllll}\mathrm{R}_{1} & \mathrm{R}_{2} & \mathrm{R}_{3} & \mathrm{R}_{4} & \mathrm{R}_{5}\end{array}$

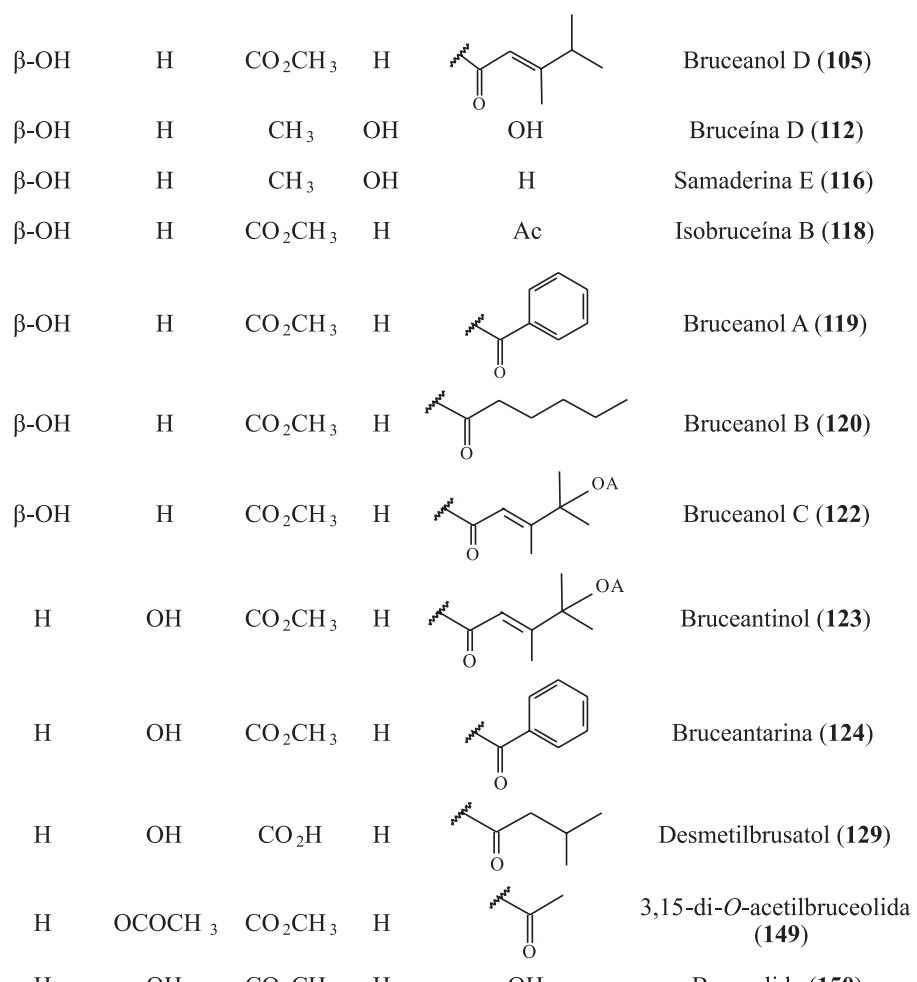

$\begin{array}{llllll}\mathrm{H} & \mathrm{OH} & \mathrm{CO}_{2} \mathrm{CH}_{3} & \mathrm{H} & \mathrm{OH} & \text { Bruceolida (150) }\end{array}$

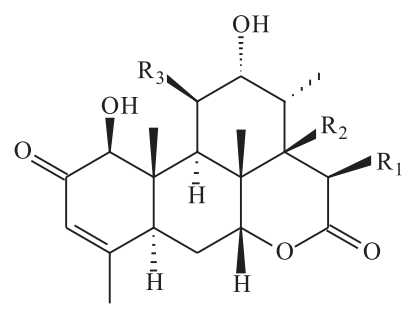

$\begin{array}{cccc}\mathrm{R}_{1} & \mathrm{R}_{2} & \mathrm{R}_{3} & \\ \mathrm{OH} & \mathrm{OH} & \mathrm{OH} & 14,15-\beta-\text { diidroxiclaineanona (109) } \\ \mathrm{H} & \mathrm{H} & =\mathrm{O} & 11-\text { deidroclai neanona }(\mathbf{1 5 7}) \\ \mathrm{OAc} & \mathrm{OH} & \mathrm{OH} & 15-\beta-O-\text {-acetil-14-hidroxiclaineanona (158) } \\ \mathrm{OH} & \mathrm{H} & \mathrm{OH} & 15-\beta-\text { hidroxiclaineanona }(159)\end{array}$

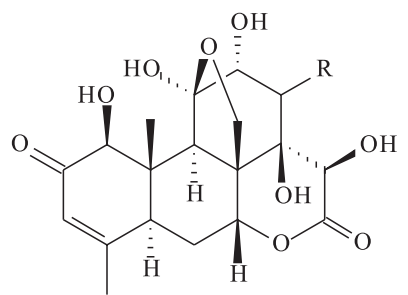

$\mathrm{R}$

$\alpha-\mathrm{CH}_{3} \quad$ Pasakbumina C (111)

B-epóxido Pasakbumina B (155)

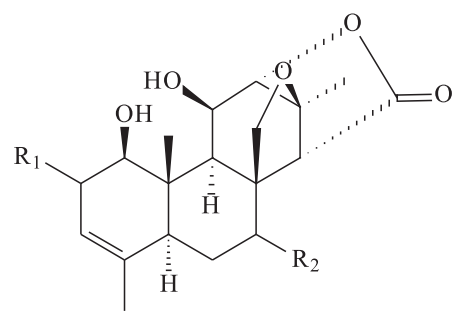

$\begin{array}{ccccc}\mathrm{R}_{1} & \mathrm{R}_{2} & \mathrm{R}_{3} & \mathrm{R}_{4} & \\ =\mathrm{O} & \alpha-\mathrm{CH}_{3} & \mathrm{OH} & \mathrm{H} & \text { 14-hidroxichaparrinona (104) } \\ =\mathrm{O} & =\mathrm{CH}_{2} & \mathrm{H} & \mathrm{C}_{4} & \\ =\mathrm{O} & =\mathrm{CH}_{2} & \mathrm{OH} & \mathrm{OH} & \text { Euricomanona (152) } \\ =\mathrm{O} & \mathrm{CH}_{3} & \mathrm{OH} & \mathrm{OH} & \text { 13,21-diidroeuricomanona (153) } \\ \alpha-\mathrm{OH} & =\mathrm{CH}_{2} & \mathrm{OH} & \mathrm{OH} & \text { Euricomanol (154) } \\ =\mathrm{O} & \alpha-\mathrm{CH}_{3} & \mathrm{H} & \mathrm{OH} & \text { Glaucarubolona (162) } \\ =\mathrm{O} & \alpha-\mathrm{CH}_{3} & \mathrm{H} & \mathrm{D}-\mathrm{Glicose} & \text { Glaucarubolona glicosilada (164) } \\ =\mathrm{O} & =\mathrm{CH}_{2} & \mathrm{H} & \mathrm{H} & \text { Ailantona (146) } \\ \alpha-\mathrm{OH} & =\mathrm{CH}_{2} & \mathrm{H} & \mathrm{H} & \text { Shinjulactona A (147) } \\ =\mathrm{O} & \alpha-\mathrm{CH}_{3} & \mathrm{H} & \mathrm{OAc} & \text { Holocantona (160) } \\ =\mathrm{O} & \alpha-\mathrm{CH}_{3} & \mathrm{H} & \mathrm{OH} & \text { Claineanona (169) }\end{array}$

Figura 8. Quassinóides que apresentam atividades biológicas destacadas

$\begin{array}{ccc}\mathrm{R}_{1} & \mathrm{R}_{2} & \\ =\mathrm{O} & =\mathrm{O} & \text { Samaderina B (114) } \\ \alpha-\mathrm{OH}, \beta-\mathrm{H} & =\mathrm{O} & \text { Samaderina C (115) } \\ \mathrm{O} & \alpha-\mathrm{OH}, \beta-\mathrm{H} & \text { Cedronina (117) } \\ \alpha-\mathrm{OH}, \beta-\mathrm{H} & \alpha-\mathrm{OH}, \beta-\mathrm{H} & \text { Cedronolina (151) }\end{array}$

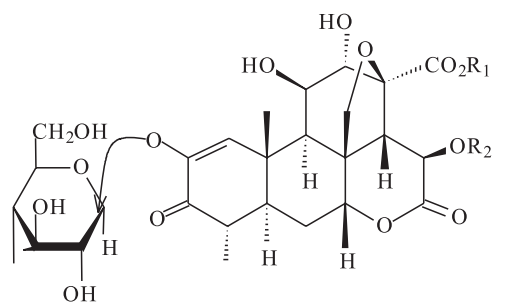

$\mathrm{R}_{1}$

$\mathrm{R}_{2}$

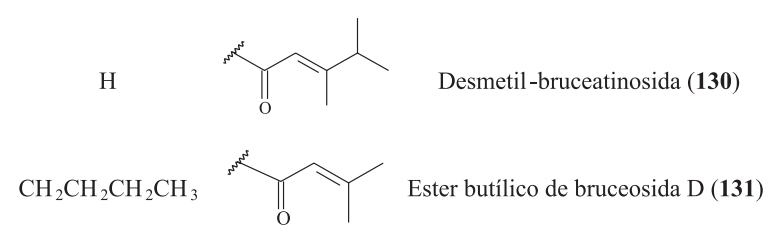




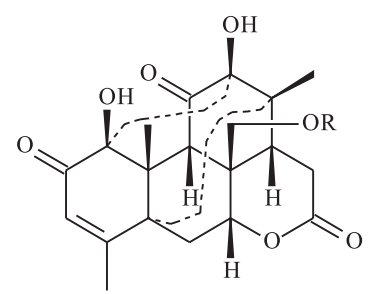

$\mathrm{R}$

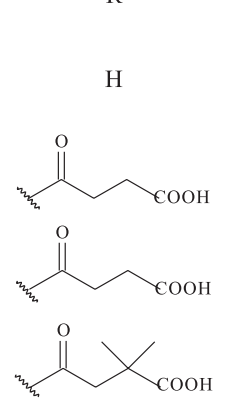

$\mathrm{R}$

Shinjulactona C

(133)

(134)

(135)

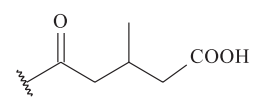

(136)<smiles>CCC(=O)CC(C)(C)CC(=O)O</smiles>

(137)<smiles>CCC(C)(CC(C)=O)CC(=O)O</smiles><smiles>CCC(=O)CC1(CC(=O)O)CCCC1</smiles>

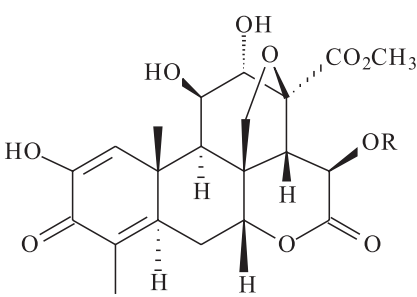

$\mathrm{R}$

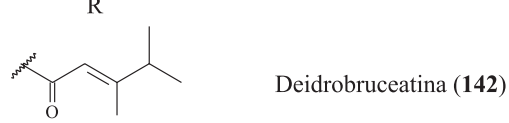

Deidrobruceantinol (143)

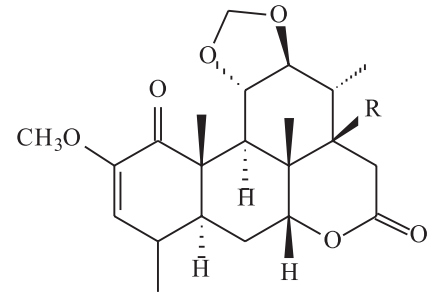

$\mathrm{R}$

H Picrasina D (144)

$\mathrm{OH}$ Picrasina E (145)

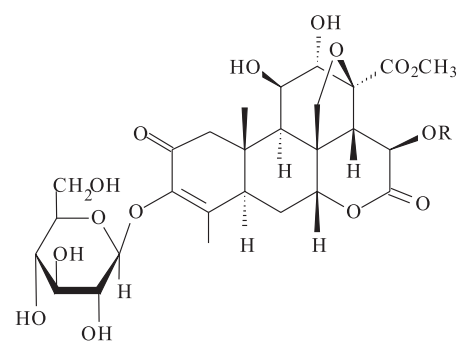

$\mathrm{R}$
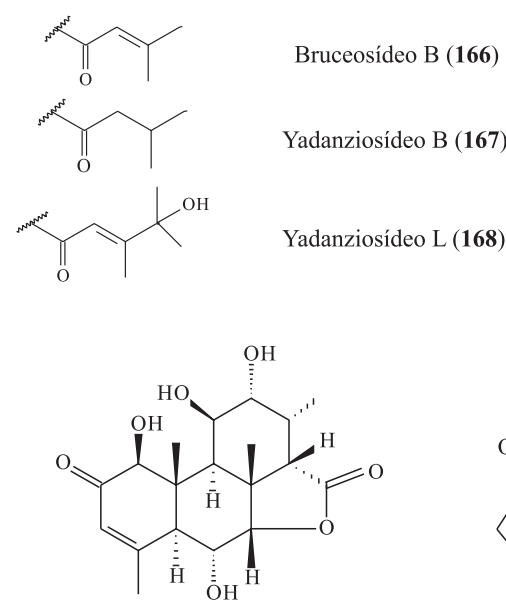

Longilactona (156)

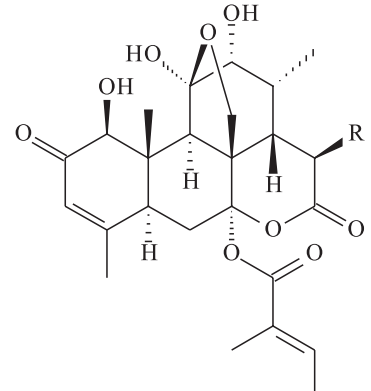

$\mathrm{R}$

$\mathrm{OH} \quad$ 15-Desacetilundulatona (113)

H 6- $\alpha$-tigloiloxichaparrinona (148)

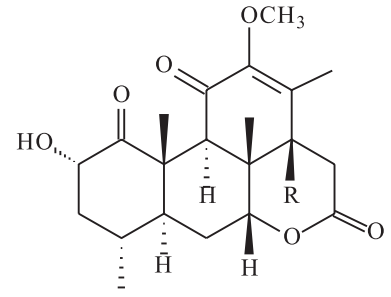

$\mathrm{R}$

H Picrasina B (161)

$\mathrm{OH} \quad$ Picrasina G (165)

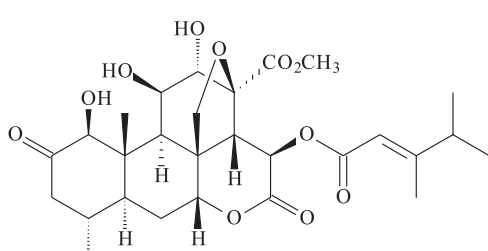

Bruceanol E (106)

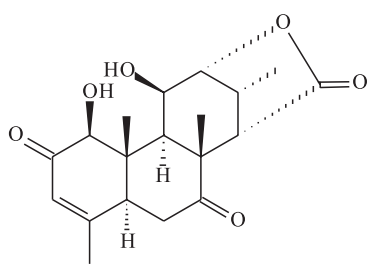

Euricomalactona (108)

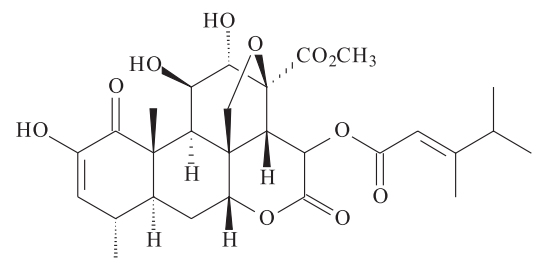

Bruceanol F (107)

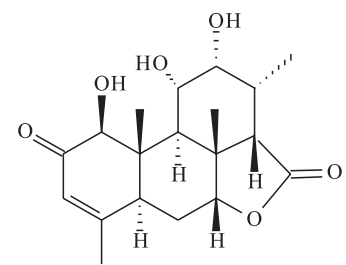

6-diidroxilongilactona (110)

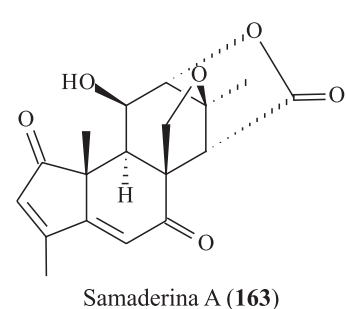

Samaderina A (163)

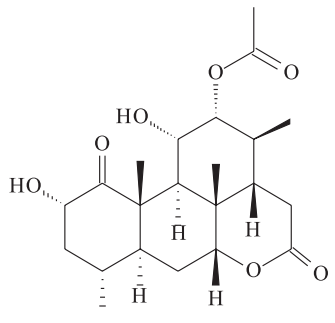

Shinjulactona K (170)

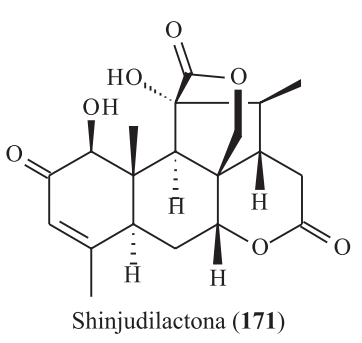

Figura 8. (continuação) 
Tabela 2. Quassinóides com atividades citotóxica e antimalárica

\begin{tabular}{|c|c|c|c|c|c|c|c|c|c|c|c|c|c|}
\hline \multirow[t]{2}{*}{ Quassinóide } & \multicolumn{4}{|c|}{$\begin{array}{c}\mathrm{CI}_{50} \\
\left(\mu \mathrm{g} \mathrm{mL} \mathrm{mL}^{-1}\right)\end{array}$} & \multirow{2}{*}{$\frac{\begin{array}{c}\mathrm{DE}_{50} \\
\left(\mu \mathrm{g} \mathrm{mL}^{-1}\right)\end{array}}{\mathrm{KB}^{\mathrm{b}}}$} & \multicolumn{2}{|c|}{$\begin{array}{c}\mathrm{DE}_{50} \\
\left(\mu \mathrm{g} \mathrm{mL} L^{-1}\right)\end{array}$} & \multirow{2}{*}{$\begin{array}{c}\mathrm{CI}_{50} \\
\left(\mu \mathrm{g} \mathrm{mL}{ }^{-1}\right) \\
\mathrm{P}-338^{\mathrm{g}}\end{array}$} & \multicolumn{3}{|c|}{$\begin{array}{c}\mathrm{DE}_{50} \\
\left(\mu \mathrm{g} \mathrm{mL} L^{-1}\right)\end{array}$} & \multirow{2}{*}{$\begin{array}{c}\begin{array}{c}\mathrm{CI}_{50} \\
\left(\mu \mathrm{gL}^{-1}\right)\end{array} \\
P . \\
\text { falciparum }\end{array}$} & \multirow[t]{2}{*}{ Ref. } \\
\hline & $\begin{array}{l}\text { SK- }^{\mathrm{a}} \\
\mathrm{MEL}\end{array}$ & $\mathrm{KB}^{\mathrm{b}}$ & $\begin{array}{l}\text { BT- } \\
549^{c}\end{array}$ & $\begin{array}{l}\text { SK- } \\
\text { OV-3 }\end{array}$ & & A- $549^{\mathrm{e}}$ & $\mathrm{MCF}-7^{\mathrm{f}}$ & & $\begin{array}{l}\text { KB- } \\
\mathrm{VIN}^{\mathrm{h}}\end{array}$ & $\begin{array}{l}\mathrm{KB}- \\
7 \mathrm{~d}^{\mathrm{h}}\end{array}$ & $\begin{array}{l}\mathrm{KB}- \\
\mathrm{CPT}^{\mathrm{h}}\end{array}$ & & \\
\hline Bruceanol A (119) & - & - & - & - & 0,0089 & - & - & - & 0,44 & 0,0081 & 0,0100 & - & 64 \\
\hline Bruceanol B (120) & - & - & - & - & 0,0077 & - & - & - & 1,00 & 0,0064 & 0,0080 & - & 64 \\
\hline Bruceanol D (105) & - & - & - & - & 0,0068 & 0,55 & - & 0,09 & 0,51 & 0,0060 & 0,0095 & - & 56,64 \\
\hline Bruceanol E (106) & - & - & - & - & 0,0035 & 3,75 & - & 0,57 & 4,40 & 0,0050 & 0,0062 & - & 56,64 \\
\hline Bruceanol F (107) & - & - & - & - & 0,43 & 0,55 & - & 0,36 & - & - & - & - & 56 \\
\hline Bruceanol G (26) & - & - & - & - & $0,334(*)$ & - & - & - & - & - & - & - & 19 \\
\hline Bruceantarina (124) & - & - & - & - & 0,038 & - & - & - & 3,50 & 0,0310 & 0,0750 & - & 64 \\
\hline Bruceantina (3) & - & - & - & - & 0,0052 & - & - & - & 0,14 & 0,0044 & 0,0066 & - & 64 \\
\hline Bruceantinol (123) & - & - & - & - & 0,0059 & - & - & - & 1,30 & 0,0062 & 0,0064 & - & 64 \\
\hline Cedronina (117) & - & 4,0 & - & - & - & - & - & - & - & - & - & 0,23 & 63 \\
\hline Chaparrinona $(\mathbf{8 8})$ & - & - & - & - & - & - & - & 0,34 & - & - & - & 0,037 & 55 \\
\hline 15-Desacetilundulatona (113) & - & - & - & - & - & - & - & - & - & - & - & 0,047 & 55 \\
\hline 13,21-Diidroeuricomanona(153) & - & 0,33 & - & - & - & - & - & - & - & - & - & 0,29 & 77 \\
\hline$\Delta^{13(18)}$-Diidroglaucarubinona(121) & - & - & - & - & 0,22 & - & - & - & 1,2 & 0,14 & 0,13 & - & 64 \\
\hline 11-Deidroclaineanona (157) & - & - & - & - & - & - & - & - & - & - & - & $1,93(*)$ & 78 \\
\hline 14,15 $\beta$-Diidroxiclaineanona(109) & - & 0,38 & - & - & - & $<2,5$ & $<2,5$ & - & - & - & - & 7,71 & 32,77 \\
\hline 6-Diidroxilongilactona (110) & - & - & - & - & - & $<2,5$ & $<2,5$ & - & - & - & - & - & 32 \\
\hline Euricomalactona (108) & - & 1,30 & - & - & - & $<2,5$ & $<2,5$ & - & - & - & - & 1,56 & 32,77 \\
\hline Euricomanol (154) & - & 3,60 & - & - & - & - & - & - & - & - & - & 3,81 & 77 \\
\hline Euricomanona (152) & - & 0,40 & - & - & - & 8,1 & $<2,5$ & - & - & - & - & 0,23 & 32,77 \\
\hline Excelsina (100) & - & - & - & - & - & - & - & - & - & - & - & $0,096(*)$ & 14 \\
\hline 14-Hidroxichaparrinona (104) & - & - & - & - & - & - & - & 0,05 & - & - & - & 0,188 & 55 \\
\hline 15- $\beta$-Hidroxiclaineanona(159) & - & - & - & - & - & - & - & - & - & - & - & $2,02(*)$ & 78 \\
\hline Indaquassina $\mathrm{C}(\mathbf{8 3})$ & - & 1,00 & - & - & - & - & - & - & - & - & - & - & 41 \\
\hline Javanicosida I (22) & - & - & - & - & - & - & - & 7,5 & - & - & - & - & 18 \\
\hline Javanicosida J (24) & - & - & - & - & - & - & - & 2,3 & - & - & - & - & 18 \\
\hline Javanicosida K (25) & - & - & - & - & - & - & - & 1,6 & - & - & - & - & 18 \\
\hline Javanicosida L (23) & - & - & - & - & - & - & - & 2,9 & - & - & - & - & 18 \\
\hline Longilactona ( 156) & - & - & - & - & - & - & - & - & - & - & - & $2,10-5,23(*)$ & 78 \\
\hline $\begin{array}{l}15-\beta-O \text {-acetil-14- } \\
\text { hidroxiclaineanona (158) }\end{array}$ & - & - & - & - & - & - & - & - & - & - & - & $10,43(*)$ & 78 \\
\hline Orinocinolida $(\mathbf{9 4})$ & 1,9 & 1,9 & 1,7 & 0,8 & - & - & - & - & - & - & - & $0,0085(*)$ & 46 \\
\hline Pasakbumina B (155) & - & - & - & - & - & 8,4 & $<2,5$ & - & - & - & - & $0,0226(*)$ & 32 \\
\hline Pasakbumina C ( 111) & - & - & - & - & - & 3,6 & $<2,5$ & - & - & - & - & $0,0934(*)$ & 32 \\
\hline Samaderina B (114) & - & 0,07 & - & - & - & - & - & - & - & - & - & $0,0760(*)$ & 41 \\
\hline Samaderina C (115) & - & 0,40 & - & - & - & - & - & - & - & - & - & - & 41 \\
\hline Samaderina E (116) & - & 0,04 & - & - & - & - & - & - & - & - & - & $0,0221(*)$ & 41 \\
\hline Samaderina X (78) & - & 0,02 & - & - & - & - & - & - & - & - & - & $0,0061(*)$ & 41 \\
\hline Samaderina Y (79) & - & 0,10 & - & - & - & - & - & - & - & - & - & - & 41 \\
\hline Samaderina Z (80) & - & 0,20 & - & - & - & - & - & - & - & - & - & $0,0280(*)$ & 41 \\
\hline Simalikalactona D (103) & 0,28 & 1,0 & 0,3 & 0,3 & - & - & - & - & - & - & - & $0,0036(*)$ & 46 \\
\hline 6- $\alpha$-Tigloiloxichaparrinona(148) & - & - & - & - & - & - & - & - & - & - & - & 0,062 & 75 \\
\hline
\end{tabular}

a - Melanoma (pele); ${ }^{\text {b }}$ Carcinoma nasofaringeal; ${ }^{\text {- }}$ Carcinoma ductal de mama; ${ }^{\mathrm{d}}$ - Carcinoma ovariano; ${ }^{\mathrm{e}}$ - Carcinoma de pulmão; ${ }^{\mathrm{f}}$ - Adenocarcinoma de mama; $\mathrm{g}$ - Leucemia linfocítica; ${ }^{\mathrm{h}}$ - Multidrogas resistentes; ${ }^{*}$ Valores convertidos para $\mu \mathrm{g} \mathrm{mL}{ }^{-1}$, para melhor comparação dos resultados

\section{Atividade antitumoral}

Já era conhecido que vários quassinóides apresentam atividade citotóxica ${ }^{1-3}$ e no período relativo a este trabalho foram registrados outros casos de quassinóides com essa atividade antitumoral ${ }^{32,52-55}$.

Dentre os quassinóides que exibiram significante atividade in vitro contra a leucemia linfocítica P-388, destaca-se cedronolactona A $(\mathbf{8 9})^{44}$, seguida, em ordem decrescente de atividade, pela 14- hidroxichaparrinona (104) e chaparrinona $(\mathbf{8 8})^{55}$. Javanicosida I (22), J (24), K (25) e L (23), quassinóides glicosilados da Brucea amarrissima mostraram moderada atividade contra P-338 ${ }^{18}$. Já Bruceanol D (105), E (106) e F (107) apresentaram potente citotoxidade in vitro em cinco linhagens de células tumorais $(\mathrm{KB}$, A-549, HCT-8, RPMI-7951 e TE-671) e ainda leucemia linfocítica $(\mathrm{P}-338)^{56}$. Os compostos euricomalactona (108), 14,15ß-diidroxiclaineanona (109), 6-diidroxilongilactona (110), pasakbumina $\mathrm{C}$ 
(111) mostraram também significante atividade contra câncer de pulmão (A-549), porém menor que os quassinóides bruceanol D e $\mathrm{F}$ e de mama MCF-7 ${ }^{32}$

A citotoxidade dos bruceosídeos D (39), E (40) e F (41) foi avaliada em 58 linhagens de células tumorais, representando 9 tipos de câncer. Estas substâncias mostraram atividade seletiva contra leucemia, melanoma, câncer ovariano, de cólon e câncer de não-pequenas células do pulmão, com $\log \mathrm{GI}_{50}$ (Log da concentração que reduz o crescimento das células em 50\%) variando de $-4,31$ a $-4,72 \mathrm{M}^{23}$.

Vários compostos foram estudados com relação à capacidade de induzir a diferenciação celular frente à leucemia promielocítica humana (HL-60), sendo que somente a bruceína D (112) (43,1\% a $\left.0,1 \mu \mathrm{g} \mathrm{mL}{ }^{-1}\right)$ apresentou significante atividade inibitória $(>40 \%)^{21}$.

O estudo da atividade de um novo quassinóide, orinocinolida (94), recentemente isolado de Simaba orinocensis, demonstrou que este composto inibia o crescimento das células cancerígenas SKMEL (pele), KB (nasofaringeal), BT-549 (mama), SK-OV-3 (ovário), porém era menos potente contra todas essas células que o conhecido simalikalactona D $(\mathbf{1 0 3})^{46}$. As atividades antimalárica ${ }^{57,58}$, anticâncer ${ }^{1,59}$ e amebicida ${ }^{54}$ de $\mathbf{1 0 3}$ foram previamente registradas e vários análogos foram sintetizados para confirmação dessas atividades biológicas ${ }^{58-60}$.

A peninsularinona (42) apresentou excelente atividade in vivo contra adenocarcinoma ductal pancreático $(\mathrm{P}-03)(4,3 \mathrm{mg} / \mathrm{kg})$ e adenocarcinoma do cólon (C-38) $(3,2 \mathrm{mg} / \mathrm{kg})$, com T/C de $3 \%{ }^{61}$.

Dentre cinco quassinóides isolados de Hannoa klaineana, somente 15-desacetilundulatona (113) mostrou significante atividade in vivo contra P-338 e C-38. A relação estrutura-atividade foi estudada, observando-se que $\mathbf{1 1 3}$ apresentava no anel A um grupo cetol nas posições 1 e 2, um grupo hidroxila livre em C-1, C-11 e $\mathrm{C}-12$, e no anel B um grupamento éster em C-6. A inatividade do outro quassinóide estudado foi atribuída à redução da carbonila em C-2. Portanto, a presença da cetona $\alpha, \beta$-insaturada é essencial para a deficiência de elétrons em $\mathrm{C}$-4, onde as funções nucleofílicas do DNA da célula tumoral poderão ser fixadas e a duplicação das células malignas, impedida ${ }^{62}$.

As samaderinas B (114), C (115), E (116), X (78), Y (79), isoladas da $Q$. indica da Indonésia, mostraram citotoxicidade in vitro $\left(\mathrm{CI}_{50}=0,04-1,00 \mu \mathrm{g} \mathrm{mL}^{-1}\right)$ contra carcinoma nasofaringeal $(\mathrm{KB})^{41}$, enquanto cedronina (117), um quassinóide de esqueleto $\mathbf{C}_{19}$, exibiu atividade relativamente baixa $\left(\mathrm{CI}_{50}=4,0 \mu \mathrm{g} \mathrm{mL} \mathrm{L}^{-1}\right)$, quando comparada com os quassinóides $\mathbf{C}_{20}$ biologicamente ativos ${ }^{63}$.

$\mathrm{O}$ tratamento quimioterapêutico bem sucedido é severamente dificultado pela resistência das multidrogas. Assim sendo, 23 quassinóides foram avaliados com relação à citotoxicidade contra 3 linhagens de células cancerígenas de multidrogas-resistentes (KBVIN, KB-7d, KB-CPT) e de carcinoma nasofaringeal com células do tipo KB. Nove compostos $(3,105,106,118-121,123,124)$ mostraram atividade significante $\left(\mathrm{DE}_{50} \leq 4 \mu \mathrm{g} \mathrm{mL}^{-1}\right)$ contra todas as 3 linhagens de multidrogas-resistentes, sendo a bruceantina (3) o mais potente (Tabela 2). Estudos da relação estrutura-atividade, baseada nesses dados, resultaram nas seguintes características necessárias para essa atividade citotóxica: uma cetona $\alpha, \beta$-insaturada e um grupo hidroxila vizinho à carbonila, no anel $\mathbf{A}$; um esqueleto básico com quatro anéis, com o anel $\mathbf{D}$ lactônico; um grupo éster em C-15 e/ou C-6. Observou-se ainda que, a troca de posição da hidroxila no $\mathrm{C}-1$ e carbonila no $\mathrm{C}-2$ reduz a atividade sensivelmente e que os quassinóides glicosilados se mostram menos ativos que suas correspondentes moléculas agliconas ${ }^{64}$.

A bruceantina (3), um quassinóide com esqueleto básico do tipo $\mathbf{C}_{20}$, muito relatado na literatura por suas atividades in vivo contra melanoma, câncer de cólon e leucemia ${ }^{53,65}$, teve sua ação comparada à do taxol e camptotecina e entrou em fase de uso clínico como candidata a fármaco anticâncer. Entretanto, nenhuma regressão objetiva do tumor foi observada e o desenvolvimento da droga foi encerrado. Recentemente, a ação da bruceantina foi novamente estudada e observou-se que esta interfere no crescimento de 11 linhagens de células leucêmicas, sugerindo que seja novamente investigada quanto a sua eficácia clínica em doenças malignas hematológicas ${ }^{53}$.

A atividade biológica de 4 quassinóides sintetizados (125-128), os quais apresentam fluoreto em C-3 ou C-15 da cadeia lateral (Tabela 3), mostrou significante potencial citotóxico contra 8 tipos de linhagens de células, incluindo o câncer de pequenas células e de não-pequenas células do pulmão, de cólon, do sistema nervoso central (SNC), do ovário, renal, leucemia e melanoma. Notadamente, a atividade citotóxica de $\mathbf{1 2 5}$ é cerca de 100 vezes mais elevada que 126 e 128. O composto 125 esterificado em C-15 com o grupo 4,4,4-trifluoro-3-metilbutanoil mostrou-se muito mais ativo que o análogo com substituinte em C-3 (128) e foi aproximadamente tão ativo quanto a bruceantina $(\mathbf{3})^{66,67}$.

Tabela 3. Citotoxidade de quassinóides fluorados

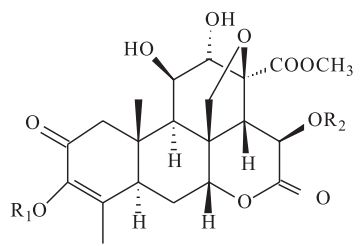

\begin{tabular}{|c|c|c|c|}
\hline Composto & $\mathrm{R}_{1}$ & $\mathrm{R}_{2}$ & $\log \mathrm{GI}_{50}$ \\
\hline Bruceantina (3) & $\mathrm{H}$ & & $-7,7--8,6$ \\
\hline (125) & $\mathrm{H}$ & & $-7,0--8,7$ \\
\hline (126) & $\mathrm{H}$ & & $-5,0--6,0$ \\
\hline (127) & & $\mathrm{H}$ & $-4,8--5,9$ \\
\hline (128) & & $\mathrm{H}$ & $-4,5--6,4$ \\
\hline
\end{tabular}

A atividade antitumoral de vários quassinóides foi também avaliada através do efeito inibitório contra a ativação do antígeno do vírus Epstein-Barr (EBV-EA) induzido por 12-O-tetradecanoilforbol-13-acetato (TPA) em células do tipo Raji ${ }^{12,68,69}$. Os quassinóides ailantinol E (8), F (9) e G (6), isolados de A. altissima ${ }^{12}$, os desmetilbrusatol (129), desmetilbruceantinosida A (130) e o éster butílico da bruceosida D (131), isolados de B. javanica $^{69}$, e os quassinóides isobruceína B (118), bruceanol A (119) e B (120), isolados da $B$. antidysenteric ${ }^{64}$, mostraram elevada atividade, sem apresentar nenhuma citotoxidade.

A shinjulactona $C$ (132), seus derivados (133-139) e a shinjulactona B (140) foram avaliados por seus efeitos quimiopreventivos contra ativação de EBV-EA. Entre todos os compostos testados, 3', 3'-dimetilsuccinato (135) derivado de 132 exibiu o maior efeito inibitório. Do ponto de vista da relação estrutura-atividade, os derivados succinatos $(\mathbf{1 3 3}, \mathbf{1 3 5})$ demonstraram maior 
atividade que os derivados glutaratos $(\mathbf{1 3 4}, \mathbf{1 3 6}, \mathbf{1 3 9})$. Como os substituintes na posição 3' tornam as moléculas mais volumosas (maior impedimento estérico), o efeito inibitório dos derivados glutaratos $(\mathbf{1 3 8}, \mathbf{1 3 9})$ decresce significativamente ${ }^{68}$.

A atividade inibitória contra a ativação de EBV-VA de 45 quassinóides das espécies $B$. antidysenterica, $B$. javanica e $P$. ailanthoides foi avaliada. Os 10 compostos mais ativos incluem bruceanol A (119), B (120), C (122), D (105), E (106), G (26), isobruceína B (118), bruceantina (3), deidrobruceantina (142) e deidrobruceantinol (143). Todos esses compostos foram isolados da $B$. antidysenterica, são agliconas contendo os grupos carbonila e hidroxila no anel A, uma ponte oximetileno entre C-8 e C-13 e um grupo éster em C-15. Dentre os menos eficientes estão quassina (1), neoquassina (2), picrasinol D (65), picrasinas D (144) e E (145). Todas essas substâncias foram obtidas de $P$. ailanthoides e não apresentam a ponte oximetilênica nem a função éster na cadeia lateral ${ }^{70}$.

Quatorze quassinóides isolados de Ailanthus altissima foram também investigados com relação a essa atividade antitumoral. Os compostos ailantinol $\mathrm{B}(\mathbf{5}), \mathrm{C}(\mathbf{6})$, ailantona (146) e shinjulactona $\mathrm{A}$ (147) mostraram moderada atividade, sendo 6 (que apresenta um grupo epóxido entre os carbonos C-12 e C-13) o quassinóide mais potente dentre os 14 estudados $^{71}$. Demais estudos da relação estrutura-atividade indicaram resultados semelhantes àqueles registrados anteriormente ${ }^{70}$.

\section{Atividade antimalárica}

A resistência criada pelo parasita da malária humana, Plasmodium falciparum, à cloroquina e outros fármacos antimaláricos tem incentivado a busca por novos fármacos para o combate à doença ${ }^{7}$. Vários quassinóides apresentaram acentuada atividade in vitro contra este parasita e existe muito registro na literatura acerca da atividade antimalárica em Simaroubaceae $e^{1,58,72,73}$. Estudos mais recentes mostram um número significativo de quassinóides com essa atividade (Tabela 2).

Os compostos samaderinas $\mathrm{X}(\mathbf{7 8}), \mathrm{Z}(\mathbf{8 0})$, E (116) e B (114) apresentam completa inibição do parasita $\left(\mathrm{CI}_{50}=0,014-0,21 \mu \mathrm{M} ; 0,0061\right.$ $\left.0,0760 \mu \mathrm{g} \mathrm{m}^{-1}\right)^{41}$. A excelsina (100) é também muito ativa $(0,2 \mu \mathrm{M}$; $0,096 \mu \mathrm{g} \mathrm{mL}^{-1}$ ), uma vez que a cloroquina (droga comercial) mostrase efetiva na concentração de $\left.0,025 \mu \mathrm{M}(0,008 \mu \mathrm{g} \mathrm{mL})^{-1}\right)^{14}$.

Testes realizados com 4 quassinóides da espécie Hannoa chorantha indicaram alto potencial antiplasmodial com valores de $\mathrm{CI}_{50}<0,1 \mu \mathrm{g} \mathrm{mL} \mathrm{mL}^{-1}$. Dois deles, chaparrinona $(\mathbf{8 8})$ e 15 -desacetilundulatona (113) tiveram valores muito mais promissores $(0,037$ e $0,047 \mu \mathrm{g} \mathrm{mL}^{-1}$, respectivamente). Chaparrinona (88) mostrou-se cinco vezes mais eficiente que a 14-hidroxichaparrinona (104) indicando, assim, que a presença da hidroxila no C-14 não favorece esta atividade ${ }^{55}$.

O quassinóide $6 \alpha$-tigloiloxichaparrinona (148), isolado pela primeira vez da A. altissima e já relatado como antineoplástico ${ }^{74}$, também apresentou elevada atividade antimalárica ${ }^{75}$.

Recentemente, foi registrado o isolamento de um novo quassinóide, a orinocinolida (94), da Simaba orinocensis, planta nativa da Amazônia com acentuada atividade antimalárica $(0,00853 \mu \mathrm{g}$ $\left.\mathrm{mL}^{-1}\right)^{46}$. Resultados promissores desta atividade in vitro levaram à pesquisa in vivo contra Plasmodium berghei. O 3,15-di-O-acetilbruceolida (149) mostrou potente atividade, com o dobro da eficiência $\left(\mathrm{DE}_{50}=0,46 \mathrm{mg} / \mathrm{kg}\right)$ daquela encontrada para a bruceolida $\left(\mathrm{DE}_{50}=1,1 \mathrm{mg} / \mathrm{kg}\right)(\mathbf{1 5 0})^{76}$.

A cedronina (117) foi avaliada com relação ao seu potencial in vitro e in vivo, mostrando-se ativa contra cloroquina-resistente, com valores de $\mathrm{CI}_{50}=0,23 \mu \mathrm{g} \mathrm{mL}{ }^{-1}$, e também contra Plasmodium vinkei, com um valor de $\mathrm{DE}_{50}$ de $1,8 \mathrm{mg} / \mathrm{kg} / \mathrm{dia}$, a uma menor concentra- ção que a dose letal ( $\left.\mathrm{DL}_{50}=10 \mathrm{mg} / \mathrm{kg} / \mathrm{dia}\right)$. Entretanto, a cedronolina (151) não mostrou significante atividade in vivo, como esperado para os quassinóides contendo grupos hidroxila ${ }^{63}$.

Estudos do potencial antiplasmodial de 5 quassinóides (108, 109, 152-154) isolados da Eurycoma longifolia e suas relações estruturaatividade mostraram que o exometileno no C-13 faz com que a euricomanona (152) tenha potência antimalárica cerca de $1,3 \mathrm{vez}$ maior que seu análogo saturado (153); os compostos 152 e 153, contendo a ponte oximetilênica entre os átomos de carbono C-8 e C-11, apresentaram-se mais eficientes que aqueles que não a possuíam $(\mathbf{1 0 8}, \mathbf{1 0 9})$ - por ex., o 153 mostrou-se cerca de 27 vezes mais potente que 109; a presença da cetona $\alpha, \beta$ insaturada no átomo de carbono C-2 do anel A, na euricomanona (152) torna o composto mais ativo que o euricomanol (154), cujo o substituinte é uma hidroxila; a euricomalactona (108), quassinóide com esqueleto $\mathrm{C}_{19}$, mostrou significativa atividade (semelhante à cloroquina), porém menor que a atividade do quassinóide $\mathrm{C}_{20}$, a euricomanona $(\mathbf{1 5 2})^{77}$.

Outros pesquisadores encontraram que a euricomanona (152) e a pasakbumina B (155), também isolados da E. longifolia, apresentaram potente atividade antimalárica contra P. falciparum $^{32}$. Entretanto, vários quassinóides (156 a 159), isolados dessa mesma espécie, mostraram atividade com $\mathrm{CI}_{50}$ entre 5,3 e 23,8 $\mu \mathrm{M}(1,93$ a $\left.10,43 \mu \mathrm{g} \mathrm{mL}^{-1}\right)$. Esses valores foram bem superiores aos registrados para outros quassinóides $\mathrm{C}_{20}$ com ponte oximetilênica entre C-8 e C-11, também encontrados E. longifolia ${ }^{78}$.

A partir dos resultados obtidos, foi possível relacionar várias características estruturais à atividade antimalárica, tais como a presença de uma hidroxila livre e o padrão de substituição e oxidação no anel A; a presença da ponte de éter entre C-8 e C-11 ou C-13 $13^{77,78}$ e, a presença de substituintes em $\mathrm{C}-15^{76}$.

\section{Atividade fitotóxica}

Os defensivos agrícolas usados no controle de ervas daninhas atingem um número limitado de sítios de ação nas moléculas ${ }^{79}$. A evolução do surgimento de ervas resistentes a muitos herbicidas comerciais tem se tornado cada vez mais problemático, porque a resistência a um herbicida pode tornar impossível o uso de outras classes de moléculas que apresentem aquele mesmo sítio de ação. Além disso, vários herbicidas comerciais têm sido removidos do mercado devido ao impacto ambiental ou ao custo de registro. Assim, produtos naturais, como os quassinóides, podem ser úteis no controle de ervas daninhas ${ }^{80}$.

Estudos demonstram que alguns extratos das raízes de Ailanthus altissima possuem aleloquímicos, os quais inibem a germinação e o crescimento de diferentes espécies, como rabanete (Raphanus sativus L.), agrião mastruço (Lepidium sativum L.) e beldroega (Portulaca oleracea L. $)^{81-83}$.

Ailantona (146), chaparrinona (88) e holocantona (160) foram identificadas como os constituintes mais ativos das espécies Ailanthus altissima, Castela tortuosa e Castela emoryi, respectivamente. Comparando-se com a atividade de herbicidas comerciais metribuzina $(500 \mu \mathrm{M})$ e trifluralina $(150 \mu \mathrm{M})$, a ailantona (146) foi o quassinóide que apresentou maior potencial fitotóxico, com a concentração de $0,9 \mu \mathrm{M}$ causando $50 \%$ de inibição em ensaios com mostarda parda (Brassica juncea) ${ }^{83,84}$.

A ailantona (146) exibiu potente atividade herbicida pré - e pós-emergência. Observando-se sua atividade pós-emergência verificou-se que baixa taxa de aplicação $(0,5 \mathrm{~kg} / \mathrm{ha})$ causou completa mortalidade de 5 dentre 7 tipos de plantas testadas, com 5 dias de tratamento. Por outro lado, alta taxa de aplicação $(8 \mathrm{~kg} / \mathrm{ha})$ não causou nenhum dano às sementes de A. altissima, indicando existir um mecanismo de proteção da espécie que previne a autotoxicidade. 
A aplicação de $\mathbf{1 4 6}$ no solo perde sua fitotoxidade, devido à atividade microbial, em 5 dias. Portanto, o alto nível de atividade herbicida pós-emergente, em conjunto com a rápida biodegradação no solo, sugere a possibilidade do uso de ailantona (146) como um herbicida natural ${ }^{82,84}$

Analisando a fitotoxicidade de 6 quassinóides, verificou-se uma importante discrepância em seus resultados. Os quassinóides do tipo picrasana (quassina (1), neoquassina (2), picrasina B (161)) mostraram-se inativos, enquanto os do tipo chaparrinona (chaparrinona $(\mathbf{8 8})$, glaucarubolona (162), holocantona (160)) apresentaram níveis elevados de fitotoxidade. Constatou-se que a ponte oximetilênica entre C-8 e C-11 em alguns quassinóides $(\mathbf{8 8}, \mathbf{1 6 2}, \mathbf{1 6 0})$ acarreta um grande efeito na conformação tridimensional, aumentando sua atividade biológica. A ausência da ponte oximetilênica observada nos quassinóides 1,2 e 161 torna estes compostos mais planares, levando a inatividade fitotóxica dessas moléculas ${ }^{80}$.

\section{Atividade fagoinibidora e inseticida}

Alguns quassinóides apresentam propriedades inseticida e fagoinibidora ("antifeedant") ou supressora de apetite em insetos. Desde o final do século XVII, a quassina (1) começou a ser usada como inseticida, com a aplicação dos extratos do caule e cascas de plantas da Quassia amara ${ }^{85}$. Estudos mais recentes demonstram essa atividade também em outras espécies e/ou outros quassinóides ${ }^{43,86,87}$.

A chaparrinona (88), isolada de uma nova espécie australiana Quassia sp. aff. bidwillii, previamente relatada por suas atividades inseticida e "antefeedant" contra lagarta do algodão (Heliothis virescens $)^{88}$, lagarta militar (Spodoptera frugiperda) e besouro do feijão mexicano (Epilachna varivestis) ${ }^{89}$, mostrou ser potencial inseticida contra as pragas aranha de duas manchas (Tetranychus urticae) e pulgão verde da batata (Myzus persicae ${ }^{43}$.

A atividade fagoinibidora contra a lagarta do fumo (Spodoptera litura) por parte dos quassinóides indaquassina $\mathrm{C}(\mathbf{8 3})$, samaderina A (163), B (114) e C(115), isolados da Samadera indica, indicaram ser 83 o mais ativo, mesmo na concentração de $0,5 \mu \mathrm{g} / \mathrm{cm}^{2}$, quando comparado com o conhecido "antifeedant" azadiractina $\mathrm{A}^{86}$ (I) (Figura 9).

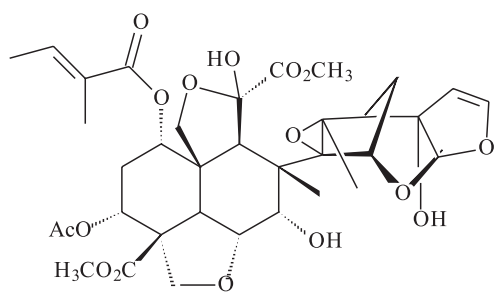

Azadiractina A (I)

Figura 9. Estrutura química da azadiractina A

A glaucarubolona glicosilada (164), isolada da Castela emoryi em plantações em terras áridas nos USA, mostrou atividade fagoinibidora contra cupins subterrâneos (Reticulitermes flavipes), espécie preocupante por acarretar prejuízo anual de bilhões de dólares ${ }^{87}$.

A atividade "antifeedant" e inseticida de 16 quassinóides de Picrasma ailanthoides foi investigada contra a larva da traça-dascrucíferas no seu $3^{\circ}$ estádio (Plutella xylostella) e a relação estrutura-atividade foi relatada. Dos compostos estudados, destacaramse quassina (1), picrasina B (161) e D (144), por seus elevados potencias "antifeedant" e inseticida, enquanto picrasina G (165) mostrou moderada atividade "antifeedant" a altas concentrações ${ }^{90}$. A traça-das-crucíferas é considerada como uma das mais impor- tantes pragas das plantas da família Brassicaceae, por acarretar elevados prejuízos, principalmente em couve e repolho ${ }^{91}$.

Dando continuidade ao estudo de atividade "antifeedant" e inseticidas contra essa importante praga agrícola, foram testados 4 quassinóides semi-sintéticos e 14 naturais ${ }^{92}$. Os resultados indicaram que os grupos metoxila em C-2 e/ou C-12 ou o grupo oximetilênico entre os carbonos C-11 e C-12 são necessários para ambas atividades e que um sistema de duplas conjugadas de C-11 a C-16 não contribui para essas atividades. Os compostos que mostraram potente atividade nas concentrações de $16-63,7 \mu \mathrm{g} / \mathrm{cm}^{2}$ apresentaram as seguintes características estruturais: uma carbonila $\alpha / \beta$ insaturada no anel A; grupos hidroxilas vicinais em $\mathrm{C}-11 \mathrm{e}$ C-12; ponte oximetilênica entre $\mathrm{C}-8$ e $\mathrm{C}-13$ e, a presença de uma cadeia lateral na posição C-15. Em seguida, esses compostos foram testados a concentrações menores de $1,0-4,0 \mu \mathrm{g} / \mathrm{cm}^{2}$ e seus resultados foram assim sumarizados: aglicona, isobruceína B (118), mostrou-se como o mais ativo "antifeedant" e inseticida; os glicosídeos, bruceosídeo B (166), yadanziosídeo B (167) e L (168), não apresentaram atividade inseticida no primeiro dia, entretanto, no segundo dia mostraram-se muito ativos. Esse resultado sugere a possibilidade da formação de agliconas através de uma gradual hidrólise dos glicosídeos ${ }^{92}$.

\section{Atividade antiparasitária}

O extrato aquoso de Castela texana, conhecida como "chaparro amargo", é tradicionalmente usado no México e China, na forma de chás ou cápsulas, no tratamento de disenterias e amebíases ${ }^{54,93,94}$. Diferentemente dos fármacos amebicidas comumente usados, o extrato aquoso de $C$. texana mostrou propriedades antimutagênicas. Isso justifica, portanto, o uso dessa planta no tratamento de amebíases $^{93}$.

Estudo da atividade in vitro contra o parasita intestinal Blastocystis hominis, em 20 tipos de extratos brutos usados na medicina tradicional chinesa, demonstrou ser o extrato de Brucea javanica um dos mais ativos $\left(500 \mu \mathrm{g} \mathrm{mL}^{-1}\right)$ dentre os extratos avaliados, quando comparado com o fármaco padrão, metronidazole $\left(10 \mu \mathrm{gL}^{-1}\right)^{95}$.

Os quassinóides longilactona (156), 11-diidroclaineanona (157) e 14,15ß-diidroxiclaineanona (109), isolados de Eurycoma longifolia, foram analisados com relação à ação esquistossomicida, usando Schistosoma japonicum. A longilactona (156) mostrou significante atividade na concentração de $200 \mu \mathrm{g} / \mathrm{mL}^{78}$.

Os nematóides fitoparasíticos que atacam principalmente as raízes das plantas estão entre as pestes agrícolas de controle mais difícil, causando sérios danos a muitas culturas de importância comercial. É necessário, portanto, o desenvolvimento de novos nematicidas. Os quassinóides chaparrinona $(\mathbf{8 8})$, claineanona (169) e glaucarubolona (162) das sementes de Hannoa undulata inibiram a ação dos nematóides causadores de galhas (Meloydogyne javanica) em tomateiros, concentração de $1-5 \mu \mathrm{g} / \mathrm{mL}^{96}$.

A ação nematicida de 38 quassinóides contra a espécie de Diplogastridae mostrou que samaderinas B (114) e E (116) foram os compostos mais ativos com a concentração letal mínima (CLM) de $2,0 \times 10^{-5} \mathrm{M}$. A atividade dos quassinóides 114 e 116, quando comparada com conhecidas drogas antiparasitárias (albendazol, tiobendazol, tiobendazol) indicou que 114 e 116 são quinze vezes mais ativos que albendazol $\left(3,0 \times 10^{-4} \mathrm{M}\right)$, dez vezes mais ativos que tiobendazol $\left(2,0 \times 10^{-4} \mathrm{M}\right)$ e sete vezes mais ativos que tiobendazol $\left(1,5 \times 10^{-4} \mathrm{M}\right)$. Portanto, samaderinas B (114) e E (116) podem ser usados como exemplares nematicidas ${ }^{97}$.

As relações entre o mecanismo de ação dos quassinóides e a morfologia das larvas têm sido estudadas e um desses mecanismos 
pode estar relacionado com o receptor ácido $\gamma$-aminobutírico $(\mathrm{GABA})^{97,98}$

\section{Outras atividades biológicas}

Um estudo da atividade antituberculose de 46 quassinóides indicou as substâncias shinjulactona $\mathrm{K}(\mathbf{1 7 0})$, ailantona (146), shinjudilactona (171) e deidrobruceantina (142) como os compostos mais ativos, embora tenham mostrado uma baixa eficiência (0$19 \%)^{99,100}$.

Os quassinóides pasakbumina A (= euricomanona) (152) e pasakbumina B (155), isolados da Eurycoma longifolia, exibiram potente atividade antiúlcera. Essa atividade foi constatada ainda na ailantona (146), quassinóide majoritário da Ailanthus altíssima, e em seu $\alpha$-epóxido ${ }^{101}$.

O potencial inibitório da replicação do vírus da imunodeficiência humana (HIV) nas células H9 linfocíticas, testado em 27 quassinóides, revelou que a shinjulactona $\mathrm{C}$ (132) mostrou ser a mais ativa $\left(\mathrm{CE}_{50}=10,6 \mu \mathrm{M}\right)$ contra o vírus $\mathrm{HIV}$, com ausência de citotoxidade $^{102}$. Investigações realizadas com quassina (1) e simalikalactona D (103) ${ }^{103}$, bem como com 19 quassinóides isolados de E. longifolia, revelaram que esses compostos são inativos contra $\mathrm{HIV}^{32}$.

A simalikalactona D (103) mostrou significante atividade antiviral contra os vírus Herpes simplex tipo 1 e Vesicular stomatitis com um fator de redução de 100 e a uma concentração de somente $0,2 \mu \mathrm{g} / \mathrm{mL}^{103}$.

Certos quassinóides apresentam também atividade antiinflamatória, como por ex., samaderinas X (78) e B(114), que mostram ainda efeito in $v i v o^{41}$.

Os quassinóides mostram ainda outras atividades, entre elas antifertilidade masculina ${ }^{104,105}$ afrodisíaco ${ }^{106-109}$, ansiolítico ${ }^{110}$ e antioxidante $^{12}$. Destacam-se a quassina (1), como o mais potente quassinóide com propriedades de antifertilidade masculina, e os ailantinol E (8) e F (9), com significativos níveis de atividade antioxidante.

\section{CONCLUSÕES}

Os quassinóides apresentam vasta gama de atividades biológicas como anticâncer, antimalárica, fitotóxica, fagoinibidora em insetos, inseticida, amebicida, nematicida, antiviral, antiúlcera, antituberculose, afrodisíaco, antifertilidade masculina, antioxidante, antiflamatória, entre outras.

Para que essas substâncias se apresentem com atividades biológicas, especialmente a citotóxica e a antimalárica, fazem-se necessárias algumas características estruturais, tais como um esqueleto de quatro anéis, com uma lactona no anel $\mathrm{D}$; uma ponte oximetileno entre $\mathrm{C}-8$ e $\mathrm{C}-11$ ou entre $\mathrm{C}-8$ e $\mathrm{C}-13$, no anel $\mathrm{C}$; uma cetona $\alpha, \beta$-insaturada no $\mathrm{C}-2$ e a presença de um grupo hidroxila livre, no anel A; um grupo éster em C-15 e/ou C-6.

A atualização bibliográfica realizada nos últimos dez anos demonstrou a presença dessas moléculas bioativas predominantemente em nove gêneros (Brucea, Eurycoma, Ailanthus, Castela, Simaba, Simarouba, Quassia, Picrasma, Picrolemma) da família Simaroubaceae. Este levantamento constatou, ainda, ser o esqueleto $\mathrm{C}_{20} \mathrm{O}$ de maior ocorrência $(76,1 \%)$ nos quassinóides pesquisados.

À vista das informações apuradas, conclui-se que os quassinóides apresentam grande variedade de atividades biológicas, constituindo um grupo de substâncias cujo estudo tem despertado muito interesse científico, pela possibilidade de larga utilização em diversas atividades agrícolas, pecuárias e, especialmente, médicas. Sugere-se, portanto, a continuidade dos estudos fitoquímicos de novos quassinóides, notadamente das Simaroubaceae, objetivando aprofundar o conhecimento dos potenciais de aplicação dessa classe de substâncias.

\section{REFERÊNCIAS}

1. Polonsky, J.; Fortschr. Chem. Org. Nat. 1985, 47, 221.

2. Grieco, P. A.; Ferriño, S.; Vidari, G.; J. Am. Chem. Soc. 1980, 102, 7586.

3. Rodrigues Filho, E.; Tese de Doutorado, Universidade Federal de São Carlos, Brasil, 1992.

4. Polonsky, J.; Fortschr. Chem. Org. Nat. 1973, 30, 101.

5. Vieira, I. J. C.; Braz Filho, R.; Rodrigues Filho, E.; Vieira, P. C.; Silva, M. F. G. F.; Fernandes, J. B.; J. Braz. Chem. Soc. 1999, 10, 76.

6. Hall, I. H.; Lee, K. H.; Imakura, Y.; Okano, M.; Johnson, A.; J. Pharm. Sci. 1983, 72, 1282.

7. Murgu, M.; Dissertação de Mestrado, Universidade Federal de São Carlos, Brasil, 1998.

8. Saraiva, R. C. G.; Pinto, A. C.; Nunomura, S. M.; Pohlit, A. M.; Quim. Nova 2006, 29, 264.

9. Jacobs, H.; Biochem. Syst. Ecol. 2003, 31, 773; Balderrama, L.; Braca, A.; Garcia, E.; Melgarejo, M.; Pizza, C.; De Tommasi, N.; Biochem. Syst. Ecol. 2001, 29, 331; Rodríguez-Gamboa, T.; Fernandes, J. B.; Rodrigues Filho, E.; Silva, M. F. G. F.; Vieira, P. C.; Ch., M. B.; Castro-Castillo, O.; Victor, S. R.; Pagnocca, F. C., Bueno, O. C.; Hebling, M. J. A.; J. Braz. Chem. Soc. 2001, 12, 386; Fernando, E. S.; Quinn, C. J.; Táxon 1995, 44, 177.

10. Kubota, K.; Fukamiya, N.; Hamada, T.; Okano, M.; Tagahara, K.; J. Nat. Prod. 1996, 59, 683.

11. Kubota, K.; Fukamiya, N.; Okano, M.; Tagahara, K.; Lee, K. H.; Bull. Chem. Soc. Jpn. 1996, 69, 3613.

12. Tamura, S.; Fukamiya, N.; Okano, M.; Koyama, J.; Koike, K.; Tokuda, H.; Aoi, W.; Takayasu, J.; Kuchide, M.; Nishino, H.; Chem. Pharm. Bull. 2003, 51,385 .

13. Joshi, B.; Pandey, A.; Sharma, R. P.; Khare, A.; Phytochemistry 2003, 62, 579.

14. Pandey, A.; Joshi, B. C.; Sharma, R. P.; Khare, A.; Med. Chem. Res. 2004, 781.

15. Aono, H.; Koike, K.; Kaneko, J.; Ohmoto, T.; Phytochemistry 1994, 37, 579.

16. Takeya, K.; Kobata, H.; Ozeki, A.; Morita, H.; Itokawa H.; J. Nat. Prod. 1997, 60, 642 .

17. Takeya, K.; Kobata, H.; Ozeki, A.; Morita, H.; Itokawa H.; Phytochemistry 1998, 48, 3, 565 .

18. Kim, I. H.; Hitotsuyanagi, Y.; Takeya, K.; Phytochemistry 2004, 65, 3167.

19. Imamura, K.; Fukamiya, N.; Nakamura, M.; Okano, M.; J. Nat. Prod. 1995, $58,1915$.

20. Kim, I. H.; Takashima, S.; Hitotsuyanagi, Y.; Hasuda, T.; Takeya, K.; J. Nat. Prod. 2004, 67, 863.

21. Su, B-N.; Chang, L. C.; Park, E. J.; Cuendet, M.; Santarsiero, B. D.; Mesecar, A. D.; Mehta, R. G.; Fong, H. H. S.; Pezzuto, J. M.; Kinghorn A. D.; Planta Med. 2002, 68, 730.

22. Kim, I. H.; Susuki, R.; Hitotsuyanagi Y.; Takeya, K.; Tetrahedron 2003, $59,9985$.

23. Ohnishi, S.; Fukamiya, N.; Okano, M.; J. Nat. Prod. 1995, 58, 1032.

24. Grieco, P. A.; Moher, E. D.; Seya, M.; Huffman, J. C.; Grieco, H. J.; Phytochemistry 1994, 37, 1451.

25. Grieco, P. A.; Haddad, J.; Piñeiro-Núñez, M. M.; Huffman, J.; Phytochemistry 1999, 50, 637.

26. Grieco, P. A.; Roest, J. M. V.; Piñeiro-Núñez, M. M.; Campaigne, E. E.; Carmack, M.; Phytochemistry 1995, 38, 1463.

27. Dou, J.; McChesney, J. D.; Sindelar, R. D.; Goins, D. K.; Walker, A.; J. Nat. Prod. 1996, 59, 73.

28. Kanchanapoom, T.; Kasai, R.; Chumsri, P.; Yamasaki, K.; Phytochemistry 2001, 57, 1205.

29. Jiwajinda, S.; Santisopasri, V.; Murakami, A.; Hirai N.; Ohigashi, H.; Phytochemistry 2001, 58, 959.

30. Ang, H. H.; Hitotsuyanagi, Y.; Takeya, K.; Tetrahedron Lett. 2000, 41, 6849.

31. Ang, H. H.; Hitotsuyanagi, Y.; Fukaya, H.; Takeya, K.; Phytochemistry 2002, 59, 833 .

32. Kuo, P-C.; Damu, A. G.; Lee, K-H.; Wu, T-S.; Bioorg. Med. Chem. 2004, $12,537$.

33. Chan, K. L.; Lee, S. P.; Sam, T. W.; Tan, S. C.; Noguchi, H.; Sankawa, U.; Phytochemistry 1991, 30, 3138.

34. Daido, M.; Fukamiya, N.; Okano, M.; J. Nat. Prod. 1995, 58, 605.

35. Novello, C. R.; Ferreira, A. G.; Marques, L. C.; Cortez, D. A. G.; Nat. Prod. Res. 2003, 17, 145.

36. Koike, K.; Yokoh, M.; Furukawa, M.; Ishi, S.; Ohmoto, T.; Phytochemistry 1995, 40, 233. 
37. Yang, S-P.; Yue, J-M.; Helv. Chim. Acta 2004, 87, 1591.

38. Zukerman-Schpector, J.; Castellano, E. E.; Rodrigues Filho, E.;Vieira, I. J. C.; Acta Crystallogr., Sect. C: Cryst. Struct. Commun. 1994, 50, 794.

39. Vieira, I. J. C.; Rodrigues-Filho, E.; Fernandes, J. B.; Silva, M. F. G. F; Vieira, P. C.; Magn. Reson. Chem. 2000, 38, 805.

40. Dou, J.; McChesney, J. D.; Sindelar, R. D.; Goins, D. K.; Khan, I. A.; Walker, L. A.; Int. J. Pharm. 1996, 34, 349.

41. Kitagawa, I.; Mahmud, T.; Yokota, K.; Nakagawa, S.; Mayumi, T.; Kobayashi, M.; Shibuya, H.; Chem. Pharm. Bull. 1996, 44, 2009.

42. Koike, K.; Ohmoto, T.; Phytochemistry 1994, 35, 459.

43. Latif, Z.; Craven, L.; Hartley, T. G.; Kemp, B. R.; Potter, J.; Rice, M. J.; Waigh, R. D.; Waterman, P. G.; Biochm. Sist. Ecol. 2000, 28, 183.

44. Ozeki, A.; Hitotsuyanagi, Y.; Hashimoto, E.; Itokawa, H.; Takeya, K.; Alves, S. M.; J. Nat. Prod. 1998, 61, 776.

45. Hitotsuyanagi, Y.; Ozeki, A.; Itokawa, H.; Alves, S. M.; Takeya, K.; J. Nat. Prod. 2001, 64, 1583.

46. Muhammad, I.; Bedir, E.; Khan, S. I.; Tekwani, B. L.; Khan, I. A.; Takamatsu, S.; Pelletier, J.; Walker, L. A.; J. Nat. Prod. 2004, 67, 772.

47. Sousa, R. P.; Barbosa, L. F.; Oliveira, A. F.; Mathias, L.; Braz Filho, R.; Vieira, I. J. C.; Nat. Prod. Res. 2005, 19, 151.

48. Arriaga, A. M. C.; Mesquita, A. C.; Pouliquen, Y. B. M.; Lima, R. A.; Cavalcante, S H.; Carvalho, M. G.; Siqueira, J. A.; Alegrio, L. V.; BrazFilho, R.; An. Acad. Bras. Ciênc. 2002, 74, 415.

49. Ghosh, P. C.; Larrahondo, J. E.; Quesne, P. W.; Raffauf, R. F.; J. Nat. Prod. 1977, 40, 364.

50. Mulholland, D. A.; Naidoo, D.; Randrianarivelojosia, M.; Cheplogoi, P. K.; Coombers, P. H.; Phytochemistry 2003, 64, 631.

51. Xu, Z.; Chang, F-R.; Wang, H-K.; Kashiwada, Y.; McPhail, A. T.; Bastow, K. F.; Tachibana, Y.; Cosentino, M.; Lee, K-H.; J. Nat. Prod. 2000, 63, 1712.

52. Cuendet, M.; Gills, J. J.; Pezzuto, J. M.; Cancer Lett. 2004, 206, 43.

53. Cuendet, M.; Pezzuto, J. M.; J. Nat. Prod. 2004, 67, 269

54. Vieira, I. J. C.; Tese de Doutorado, Universidade Federal de São Carlos, Brasil, 1995.

55. François, G.; Diakanamwa, C.; Timperman, G.; Bringmann, G.; Steenackers, T.; Atassi G.; Looveren, M. V.; Holenz, J.; Tassin, J-P.; Assi, L. A.; VanhaelenFastré, R.; Vanhaelen, M.; Int. J. Parasitology 1998, 28, 635.

56. Imamura, K.; Okano, M.; Fukamiya, N.; Tagahara, K.; Lee, K.; J. Nat. Prod. 1993, 56, 2091

57. Cabral, J. A.; McChesney, J. D.; Milhous, W. K.; J. Nat. Prod. 1993, 56, 1954.

58. Trager, W.; Polonsky, J.; J. Am. Trop. Med. Hyg. 1981, 30, 531

59. Pezzuto, J. M.; McChesney, J. D.; Cuendet, M. A.; Helson, L.; US pat 2003149096, 2003.

60. Grieco, P. A.; Morre, D. J.; Corbett, T. H.; Valeriote, F. A.; CAN pat. 125:105154, 1996

61. Moher, E. D.; Reilly, M.; Grieco, P. A.; Corbett, T. H.; Valeriote, F. A.; J. Org. Chem. 1998, 63, 3508.

62. Lumonadio, L.; Atassi, G.; Vanhaelen, M.; Fastre, R. V.; J. Ethnopharmacol. 1991, 31, 59

63. Moretti, C.; Deharo, E.; Sauvain, M.; Jardel, C.; David, P. T.; Gasquet, M.; J. Ethnopharmacol. 1994, 43, 57.

64. Murakami, C.; Fukamiya, N.; Tamura, S.; Okano, M.; Bastow, K. F.; Tokuda, H.; Mukainaka, T.; Nishino, H.; Lee, K-H.; Bioorg. Med. Chem. 2004, 12, 4963 .

65. Kupchan, S. M.; Britton, R. W.; Ziegler, M. F.; Sigel, C. W.; J. Org. Chem. 1973, 38, 178.

66. Ohno, N.; Fukamiya, N.; Okano, M.; Tagahara, K.; Lee, K-H.; Bioorg. Med. Chem. 1997, 5, 1489.

67. Lee, K-H.; Med. Res. Rev. 1999, 19, 569.

68. Tamura, S.; Fukamiya, N.; Okano, N.; Tokuda, H.; Aoi, W.; Mukainaka, T.; Nishino, H.; Tagahara, K.; Koike, K .; Cancer Lett. 2002, 185, 47.

69. Rahman, S.; Fukamiya, N.; Tokuda, H.; Nishino, H.; Tagahara, K.; Lee, K-H.; Okano, M.; Bull. Chem. Soc. Jpn. 1999, 72, 751.

70. Okano, M.; Fukamiya, N.; Tagahara, K.; Tokuda, H.; Iwashima, A.; Nishino, H.; Lee, K-H.; Cancer Lett. 1995, 94, 139.

71. Kubota, K.; Fukamiya, N.; Tokuda, H.; Nishino, H.; Tagahara, K.; Lee, K-H.; Okano, M..; Cancer Lett. 1997, 113, 165.

72. O'Neill, M. J.; Bray, D. H.; Boaedman, P.; Phillipson, J. D.; Warhurst, D. C.; Peters, W.; Suffness, M.; Antimicrob. Agents Chemother. 1986, 30, 101
73. Ang, H. H.; Chan, K. L.; Mak, J. W.; Planta Med. 1995, 61, 177.

74. Arisawa, M.; Kinghorn, A. D.; Cordel, G. A.; Farnsworth, N. R.; J. Nat Prod. 1983, 46, 218

75. Okunade, A, L.; Bikoff, R. E.; Casper, S. J.; Oksman, A.; Goldberg, D. E.; Lewis, W. H.; Phytother. Res. 2003, 17, 675.

76. Kim, H-S.; Shibata, Y.; Ko, N.; Ikemoto, N.; Ishizuka, Y.; Murakami, N.; Sugimoto, M.; Kobayashi, M.; Wataya, Y.; Parasitol. Int. 2000, 48, 271.

77. Chan, K-L.; Choo, C-Y.; Abdullah, N. R.; Ismail, Z.; J. Ethnopharmacol. 2004, 92, 223.

78. Jiwajnda, S.; Santisopasri, V.; Murakami, A.; Kawanaka, H.; Grasquet, M. Eilas, R.; Balansard, G.; Ohigashi, H.; J. Ethnopharmacol. 2002, 82, 55.

79. Duke, S. O.; Dayan, F. E.; Hermández, A.; Duke, M. V.; Abbas, H. K.; Proc. Brighton Crop Protect. Conf. 1997, 579.

80. Dayan, F. E.; Watson, S. B.; Galindo, J. C. G.; Hernández, A.; Dou, J.; McChesney, J. D.; Duke, S. O.; Pestic. Biochem. Physiol. 1999, 65, 15.

81. Feo, V.; Martino, L.; Quaranta, E.; Pizza, C.; J. Agric. Food Chem. 2003, $51,1177$.

82. Heisey, R. M.; Am. J. Bot. 1996, 83, 192

83. Heisey, R. M.; Heisey,T. K.; Plant Soil 2003, 256, 85.

84. Lin, L-J.; Peiser, G.; Ying, B-P.; Mathias, C.; Karasina, F.; Wang, Z.; Itatani, J.; Green, L.; Hwang, Y-S.; J. Agric. Food Chem. 1995, 43, 1708.

85. Viegas Jr., C.; Quim. Nova 2003, 26, 390; Simões, C. M. O.; Schenkel, E. P.; Gosmann, G.; Mello, J. C. P.; Mentz, L. A.; Petrovick, P. R.; Farmacognosia - da planta ao medicamento, $5^{\mathrm{a}}$ ed., Ed. Universidade/ UFRGS/Ed. UFSC: Porto Alegre/Florianópolis, 2004, cap. 35.

86. Govindachari, T. R.; Kumari, G. N. K.; Gopalakrishnan, G.; Suresh, G.; Wesley, S. D.; Sreelatha, T.; Fitoterapia 2001, 72, 568

87. Gutiérrez, C.; Gonzalez-Coloma, A.; Hoffmann, J. J.; Ind. Crop. Prod. 1999, 10, 35.

88. Klocke, J. A.; Arisawa, M.; Handa, S. S.; Kinghorn, A. D.; Cordel, G. A.; Farnsworth, N. R.; Experientia 1985, 41, 379.

89. Leskinen, V.; Polonsky, J.; Bhatnagar, S.; J. Chem. Ecol. 1984, 10, 1497.

90. Daido, M.; Fukamiya, N.; Okano, M.; Tagahara, K.; Hatakoshi, M.; Yamazaki, H.; Biosci., Biotechnol., Biochem. 1993, 57, 244.

91. Monnerat, R, G.; Leal-Bertioli, S. C. M.; Bertioli, D. J.; Butt, T. M.; Bordat, D.; Hortic. Bras. 2004, 22, 607.

92. Daido, M.; Ohno, N.; Imamura, K.; Fukamiya, N.; Hatakoshi, M.; Yamazaki, H.; Tagahara, K.; Lee, K-S.; Okano, M.; Biosci., Biotechnol., Biochem. 1995, 59, 974.

93. Reyes-López, M.; Villa-Treviño, S.; Arriaga-Alba, M.; Alemán-Lazarini, L.; Rodriguez-Mendiola, M.; Arias-Castro, C.; Fattel-Fazenda, S.; Garza, M.; Toxicol. in Vitro 2005, 19, 91.

94. Heinrich, M.; Kuhnt, M.; Wright, C. W.; Rimpler, H.; Phillipson, J. D.; Schandelmaier, A.; Warhurst, D. C.; J. Ethnopharmacol. 1992, 36, 81

95. Yang, L. Q.; Singh, M.; Yap, E. H.; Ng, G. C.; Xu, H. X.; Sim, K. Y.; $J$. Ethnopharmacol. 1996, 55, 35.

96. Chitwood, D. J.; Annu. Rev. Phytopathol. 2002, 40, 221.

97. Watanabe, I.; Koike, K.; Satou, T.; Nikaido, T.; Biol. Pharm. Bull. 2000, 23,723 .

98. Kuriyama, T.; Ju, X-L.; Fusazaki, S.; Hishinuma, H.; Satou, T.; Koike, K.; Nikaido, T.; Ozoe, Y.; Pestic. Biochem. Physiol. 2005, 81, 176.

99. Rahman, S.; Fukamiya, N.; Okano, M.; Tagahara, K.; Lee, K-H.; Chem. Pharm. Bull. 1997, 45, 1527.

100. Newton, S.; Lau, C.; Wright, C. W.; Phytother. Res. 2000, 14, 303.

101. Tada, H.; Yasuda, F.; Otani, K.; Doteuchi, M.; Ishihara, Y.; Shiro, M.; Eur. J. Med. Chem. 1991, 26, 345

102. Okano, M.; Fukamiya, N.; Tagahara, K.; Cosentino, M.; Lee, T. T-Y. Morris-Natschke, S.; Lee, K-H.; Bioorg. Med. Chem. Lett. 1996, 6, 701.

103. Apers, S.; Cimanga, K.; Berghe, D. V.; Meenen, E. V.; Longanga, A. O.; Foriers, A.; Vlientinck, A.; Pieters, L.; Planta Med. 2002, 68, 20.

104. Parveen, S.; Das, S.; Kundra, C. P.; Pereira, B. M. J.; Reprod. Toxicol. 2003, $17,45$.

105. Raji, Y.; Bolarinwa, A. F.; Life Sci. 1997, 61, 1067.

106. Ang, H. H.; Sim, M. K.; Exp. Anim. 1997, 46, 287.

107. Ang, H. H.; Sim, M. K.; Biol. Pharm. Bull. 1998, 21, 153.

108. Ang, H. H.; Ikeda, S.; Gan, E. K.; Phytother. Res. 2001, 15, 435.

109. Ang, H. H.; Lee, K. L.; Fundam. Clin. Pharmacol. 2002, 16, 479.

110. Ang, H. H.; Cheang, H. S.; Jpn. J. Pharmacol. 1999, 79, 497. 